medRxiv preprint doi: https://doi.org/10.1101/2021.02.24.21252329; this version posted February 26, 2021. The copyright holder for this preprint (which was not certified by peer review) is the author/funder, who has granted medRxiv a license to display the preprint in It is made available under a CC-BY-ND 4.0 International license.

1 Running head: Long COVID-19 and cognition

2

3

\title{
Long COVID neuropsychological deficits after severe, moderate or mild infection
}

P. Voruz ${ }^{1,2,3}$, G. Allali ${ }^{2,3}$, L. Benzakour ${ }^{3,4}$, A. Nuber-Champier ${ }^{1}$, M. Thomasson ${ }^{1,2}$, I. Jacot The, $^{1,2}$,

J. Pierce ${ }^{1}$, P. Lalive ${ }^{2,3}$, K-O. Lövblad ${ }^{3,5}$, O. Braillard ${ }^{6}$, M. Coen ${ }^{3,7}$, J. Serratrice ${ }^{3,7}$, J. Pugin ${ }^{3,8}$,

\# These authors contributed equally to this work

Affiliations:

${ }^{1}$ Clinical and Experimental Neuropsychology Laboratory, Faculty of Psychology, University of Geneva, Geneva, Switzerland

${ }^{5}$ Diagnostic and Interventional Neuroradiology Department, Geneva University Hospitals,

19 Switzerland

$20{ }^{6}$ Division and Department of Primary Care, Geneva University Hospitals, Switzerland

$21{ }^{7}$ Internal Medicine Department, Geneva University Hospitals, Switzerland

$22{ }^{8}$ Intensive Care Department, Geneva University Hospitals, Switzerland

$23{ }^{9}$ Neurorehabilitation Department, Geneva University Hospital, Switzerland

2410 Rhinology-Olfactology Unit, Otorhinolaryngology Department, Geneva University 25 Hospitals, Switzerland

*Corresponding author:

28 Dre Julie Péron, Faculté de Psychologie et des Sciences de l'Education, 40 bd du Pont d'Arve, 1205 Geneva, Switzerland, Tel.: +4122379 9455

30 julie.peron@unige.ch 
medRxiv preprint doi: https://doi.org/10.1101/2021.02.24.21252329; this version posted February 26, 2021. The copyright holder for this preprint (which was not certified by peer review) is the author/funder, who has granted medRxiv a license to display the preprint in It is made available under a CC-BY-ND 4.0 International license.

Voruz et al. - Long COVID-19 and cognition

ABSTRACT

Background: There is growing awareness that severe acute respiratory syndrome coronavirus 2 (SARS-CoV-2) infection can include long-term neuropsychological deficits, even in its mild or moderate respiratory forms.

Methods: Standardized neuropsychological, psychiatric, neurological and olfactory tests were administered to 45 patients (categorized according to the severity of their respiratory symptoms during the acute phase) $236.51 \pm 22.54$ days post-discharge following SARS-CoV-2 infection.

Results: Deficits were found in all the domains of cognition and the prevalence of psychiatric symptoms was also high in the three groups. The severe performed more poorly on long-term episodic memory and exhibited greater anosognosia. The moderate had poorer emotion recognition, which was positively correlated with persistent olfactory dysfunction. The mild were more stressed, anxious and depressed. neuropsychological phenotypes. 
medRxiv preprint doi: https://doi.org/10.1101/2021.02.24.21252329; this version posted February 26, 2021. The copyright holder for this preprint (which was not certified by peer review) is the author/funder, who has granted medRxiv a license to display the preprint in it is made available underpetuity.

Voruz et al. - Long COVID-19 and cognition

\section{INTRODUCTION}

The presence of long-term neuropsychological deficits following severe acute respiratory syndrome coronavirus 2 (SARS-CoV-2) infection is strongly suspected, even in its mild or moderate forms. This is based on four main arguments.

First, longitudinal studies of SARS-CoV and the Middle East respiratory syndrome, which share many pathogenetic similarities with SARS-CoV-2, have demonstrated the presence of sleep disorders, frequent recall of traumatic memories, emotional lability, impaired concentration, fatigue, and impaired memory in more than $15 \%$ of affected patients 1 month to 3.5 years following infection (Rogers et al., 2020).

Second, neurological and cognitive symptoms observed in $38.6 \%$ of patients in the acute phase (Mao et al., 2020) are hypothesized to have similar pathophysiological causes to those responsible for short- and long-term cognitive impairment in other pathologies.

Neuropsychological studies among patients with neuro-immunological diseases such as HIV (Wendelken \& Valcour, 2012), multiple sclerosis (Piras et al., 2003), and encephalitis (van Sonderen et al., 2016), have reported specific long-term deficits in cognitive functions (e.g., memory, executive or emotional processes) with a neuro-infectious and neuro-immunological pathogenesis. Furthermore, increased prevalence of stroke has been reported in patients with COVID-19 (Merkler et al., 2020; Nannoni, de Groot, Bell, \& Markus, 2020), leading to additional short- and long-term neurological and cognitive deficits, depending on the location of the lesion, as described for example by Oxley et al. (2020), who examined five patients aged under 50 years with large-vessel stroke.

Third, sudden-onset anosmia is a symptom that has been described extremely frequently by patients following infection with SARS-CoV-2, regardless of the severity of their respiratory symptoms (De Maria, Varese, Dentone, Barisione, \& Bassetti, 2020; Lee \& Lee, 2020). Researchers have identified sustentacular cells as the potential entry point into the 
medRxiv preprint doi: https://doi.org/10.1101/2021.02.24.21252329; this version posted February 26, 2021. The copyright holder for this preprint (which was not certified by peer review) is the author/funder, who has granted medRxiv a license to display the preprint in perpetuity.

Voruz et al. - Long COVID-19 and cognition

73 olfactory epithelium (Brann et al., 2020). Unlike olfactory neurons, these cells carry ACE2

receptors (Fodoulian et al., 2020). However, the exact extent to which the olfactory

epithelium is affected is still unclear, so it is currently impossible to predict which patients

not known if and how olfactory neurons are affected by disruption of sustentacular cell

function. It is also unclear whether the SARS-CoV-2 infection is confined solely to the olfactory epithelium (Vaira et al., 2020), or whether it follows a neuroinvasive pathway via the cribriform plate. Based on other neuro-olfactory pathologies, it has been suggested that entry through the nose-brain barrier is likely and probably underestimated (Doty, 2008;

Landis, Vodicka, \& Hummel, 2010). Some authors suggest that the olfactory bulb is damaged following COVID-19 infection (Kandemirli, Altundag, Yildirim, Sanli, \& Saatci, 2021; Meinhardt et al., 2021). Interestingly, an ${ }^{18}$ F-FDG PET study among patients with SARSCoV-2 and anosmia highlighted hypometabolism specifically in the neural substrates of the olfactory circuit, which could indicate an attack on the central nervous system (CNS) (pre/postcentral gyrus, thalamus/hypothalamus, cerebellum and brainstem) via the olfactory pathway (Guedj et al., 2021; Guedj et al., 2020).

Fourth, to our knowledge only one study has so far explored the short-term impact (10-40 days post-hospital discharge) of SARS-CoV-2 infection on cognition using a validated and standardized methodology with face-to-face interviews (Almeria, Cejudo, Sotoca, Deus, \& Krupinski, 2020). These authors reported short-term disruption of memory, attention, and executive functions. Unfortunately, they did not explore the impact of the severity of the respiratory symptoms. Hampshire et al. (2020) did consider the influence of severity in their study, but only found a trend toward significance, and used online tests that had not been psychometrically validated. Woo et al. (2020) also addressed the short-term (20-105 days post-infection) impact of SARS-CoV-2 in mild or moderate patients, by administering the 
medRxiv preprint doi: https://doi.org/10.1101/2021.02.24.21252329; this version posted February 26, 2021. The copyright holder for this preprint (which was not certified by peer review) is the author/funder, who has granted medRxiv a license to display the preprint in perpetuity.

Voruz et al. - Long COVID-19 and cognition

98 Modified Telephone Interview for Cognitive Status, a screening battery that was initially

99 developed for the early detection of dementia. They reported memory and attentional deficits

100 in patients compared with matched controls. These approaches had several potential

101 methodological issues, such as the use of an online survey relying on participants' unverified

102 self-reports (Hampshire et al., 2020), and the failure to collect information about patients'

103 clinical history or medical antecedents (Almeria et al., 2020; Woo et al., 2020), which may

104 have induced interindividual variability in the results. Moreover, no study has investigated the

105 long-term effects of infection on the instrumental domains (including visuospatial processing,

106 ideomotor praxis, and language) or emotion recognition. Finally, to our knowledge, the

107 impact of psychiatric factors on the cognitive functioning of patients with SARS-CoV-2 has

108 not been studied thus far. Epidemiological studies have highlighted the impact of the

109 pandemic and related health measures such as lockdown on mental health (Bäuerle et al.,

110 2020; Röhr et al., 2020; Salari et al., 2020), with increased anxiety and depressive symptoms

111 (Wang et al., 2020) within the general population. Being infected by SARS-CoV-2 also has a

112 major affective impact (Almeria et al., 2020). Long-term psychiatric consequences of

113 COVID-19 described so far include anxiety, depressive symptoms, insomnia, and

114 posttraumatic stress disorder (PTSD) (Mazza et al., 2020), especially among patients with a

115 history of psychiatric illness or who required intensive care. All these symptoms may arise

116 from a neurobiological disturbance and the ensuing neuroinflammation process (Kohler,

117 Krogh, Mors, \& Eriksen Benros, 2016).

118 In this context, the present study had three main objectives: i) investigate whether

119 SARS-COV-2 causes long-term (6-9 months after the acute phase) neuropsychological

120 deficits, identify the nature of the affected cognitive and psychiatric domains, and determine

121 their impact on quality of life; ii) explore whether cognitive and psychiatric symptoms are a

122 function of the severity of the respiratory symptoms in the acute phase, and whether patients 
medRxiv preprint doi: https://doi.org/10.1101/2021.02.24.21252329; this version posted February 26, 2021. The copyright holder for this preprint (which was not certified by peer review) is the author/funder, who has granted medRxiv a license to display the preprint in

Voruz et al. - Long COVID-19 and cognition

123 who present with moderate or even mild forms also exhibit cognitive dysfunctions and/or

psychiatric symptoms; and iii) look for correlations between long-term neuropsychological

deficits and psychiatric symptoms resulting from a neurobiological disturbance caused by

SARS-CoV-2 and/or personal stressful experience in the context of the global health crisis,

but also between these deficits and olfactory functions. To this end, patients underwent a

comprehensive neuropsychological assessment probing multiple cognitive domains, emotion

(not hospitalized). affect patients' functioning and quality of life 6-9 months post-infection. We expected to observe cognitive deficits in memory, executive function and logical reasoning (Almeria et al., 2020), as well as the emergence of psychiatric disorders such as anxiety, depressive symptoms, insomnia, and PTSD (Troyer, Kohn, \& Hong, 2020; Varatharaj et al., 2020).

139 Second, we hypothesized that the presence of neuropsychological deficits is positively 140 correlated with disease severity in the acute phase (Hampshire et al., 2020). Third, although

141 ours was an exploratory study, we hypothesized that pandemic- and disease-related

142 psychiatric symptoms explain a significant proportion (but not all) of the variance observed

143 for neuropsychological measures (Baig, Khaleeq, Ali, \& Syeda, 2020). Based on Soudry,

144 Lemogne, Malinvaud, Consoli, and Bonfils (2011) and Guedj et al. (2021), we also predicted

145 that a long-term reduction in olfactory performance would correlate positively with any

146 impaired performance on memory and emotion recognition, owing to common neuronal

147 substrates. 
medRxiv preprint doi: https://doi.org/10.1101/2021.02.24.21252329; this version posted February 26, 2021. The copyright holder for this preprint (which was not certified by peer review) is the author/funder, who has granted medRxiv a license to display the preprint in perpetuity.

Voruz et al. - Long COVID-19 and cognition

\section{METHODS}

149 Participants (see Table 1)

150 Three groups of patients who had been infected with SARS-CoV-2 were included in the

151 study: 15 patients who had been admitted to intensive care during the acute phase of the

152 infection (severe); 15 patients who had been hospitalized but did not require intensive care

153 (moderate); and 15 patients who had tested positive but had not been hospitalized. All the

154 patients had had their infection confirmed by positive polymerase chain reaction (PCR) from

155 nasopharyngeal swab and/or positive serology. On average, the moderate patients had been

156 hospitalized for 9.27 days $( \pm 9.52)$, and the severe patients for 37.40 days $( \pm 30.50)$. In

157 comparison with other studies on SARS-CoV-2, the mean duration of hospitalization for the

158 moderate group was somewhat longer, but this was driven by a single patient. The median

159 number of days for this group was 7 , which is comparable to that observed in other studies in

160 Switzerland (Regina et al., 2020).

The required number of participants in each group was determined by a power

162 analysis involving the comparison of two means: $N=\frac{2 \times \sigma^{2}\left(\frac{z \alpha}{2}+z_{\beta}\right)}{\left(\overline{\mathrm{X}}_{1}-\overline{\mathrm{X}}_{2}\right)^{2}}$. This analysis was based

163 on the literature evaluating the short-term neuropsychological effects of SARS-CoV-2 on

164 mild patients (Woo et al., 2020). To achieve the desired statistical power $(1-\beta)$ of $90 \%$ and

165 risk of Type I error $(\alpha)$ of 0.05 , results indicated that for a one-sided hypothesis, 13

166 participants were needed in each group. As we planned to perform nonparametric analyses,

167 we had to increase the sample size by $15 \%$ (Lehmann, 2012), resulting in 15 participants per

168 group.

The three groups were matched for median age $($ mild $=57$ years; moderate $=55$ years;

170 severe $=59$ years $)$, sociocultural level, and clinical variables. Given the risk factors associated

171 with the severe form of SARS-CoV-2, there were significantly higher proportions of men 
medRxiv preprint doi: https://doi.org/10.1101/2021.02.24.21252329; this version posted February 26, 2021. The copyright holder for this preprint (which was not certified by peer review) is the author/funder, who has granted medRxiv a license to display the preprint in It is made available under a CC-BY-ND 4.0 International license.

Voruz et al. - Long COVID-19 and cognition

$172 \quad($ severe $=86.66 \% ;$ moderate $=40 \% ;$ mild $=46.66 \%)$ and patients with diabetes. Participants

173 were recruited via admission lists provided by the treating doctors at Geneva University

174 Hospitals: LB and OB. For each patient, we carried out a medical file review, followed by a

175 telephone call inviting the patient to take part in the study, if all the eligibility criteria were

176 met. Exclusion criteria were a history of neurological issues, psychiatric disorders (two of the

177 included participants had had an episode of depression more than 10 years before their SARS-

178 CoV-2 infection), cancer (to exclude possible chemotherapy- and radiotherapy-related

179 cognitive impairment (Cascella et al., 2018)), neuro-developmental pathologies, pregnancy,

180 and age above 80 years. 
medRxiv preprint doi: https://doi.org/10.1101/2021.02.24.21252329; this version posted February 26, 2021. The copyright holder for this preprint (which was not certified by peer review) is the author/funder, who has granted medRxiv a license to display the preprint in It is made available under a CC-BY-N.

Voruz et al. - Long COVID-19 and cognition

181 Table 1: Sociodemographic data and relevant medical antecedents

182

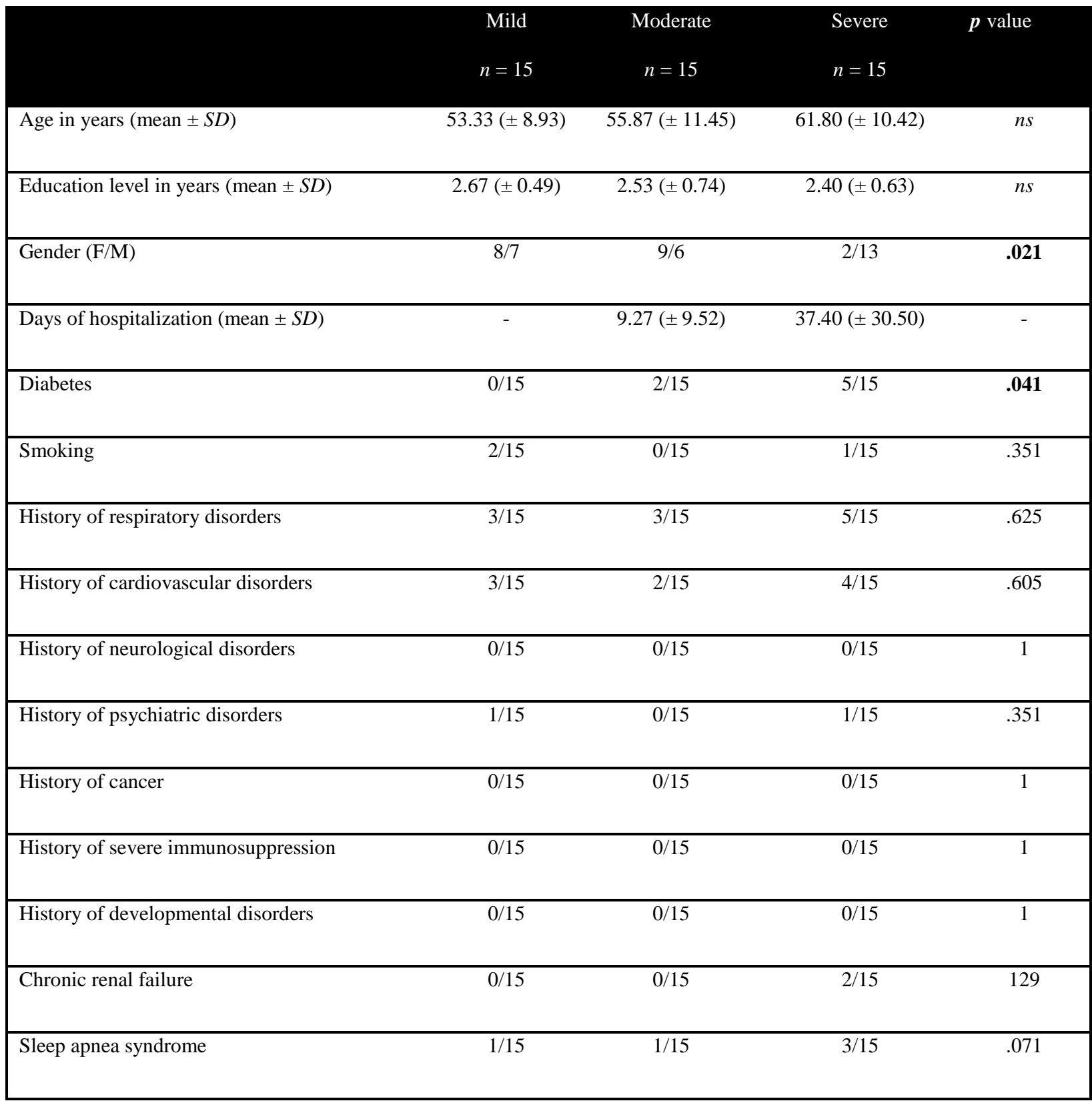

183

184 Note. F: female; M: male; ns: not significant; SD: standard deviation. 
medRxiv preprint doi: https://doi.org/10.1101/2021.02.24.21252329; this version posted February 26, 2021. The copyright holder for this preprint (which was not certified by peer review) is the author/funder, who has granted medRxiv a license to display the preprint in it is merpetuity.

Voruz et al. - Long COVID-19 and cognition

\section{General procedure and ethics}

186 A flowchart displaying the successive stages of the study according to the eligibility criteria

187 for each experimental group is provided in Figure 1.

After being given a complete description of the study, participants provided their

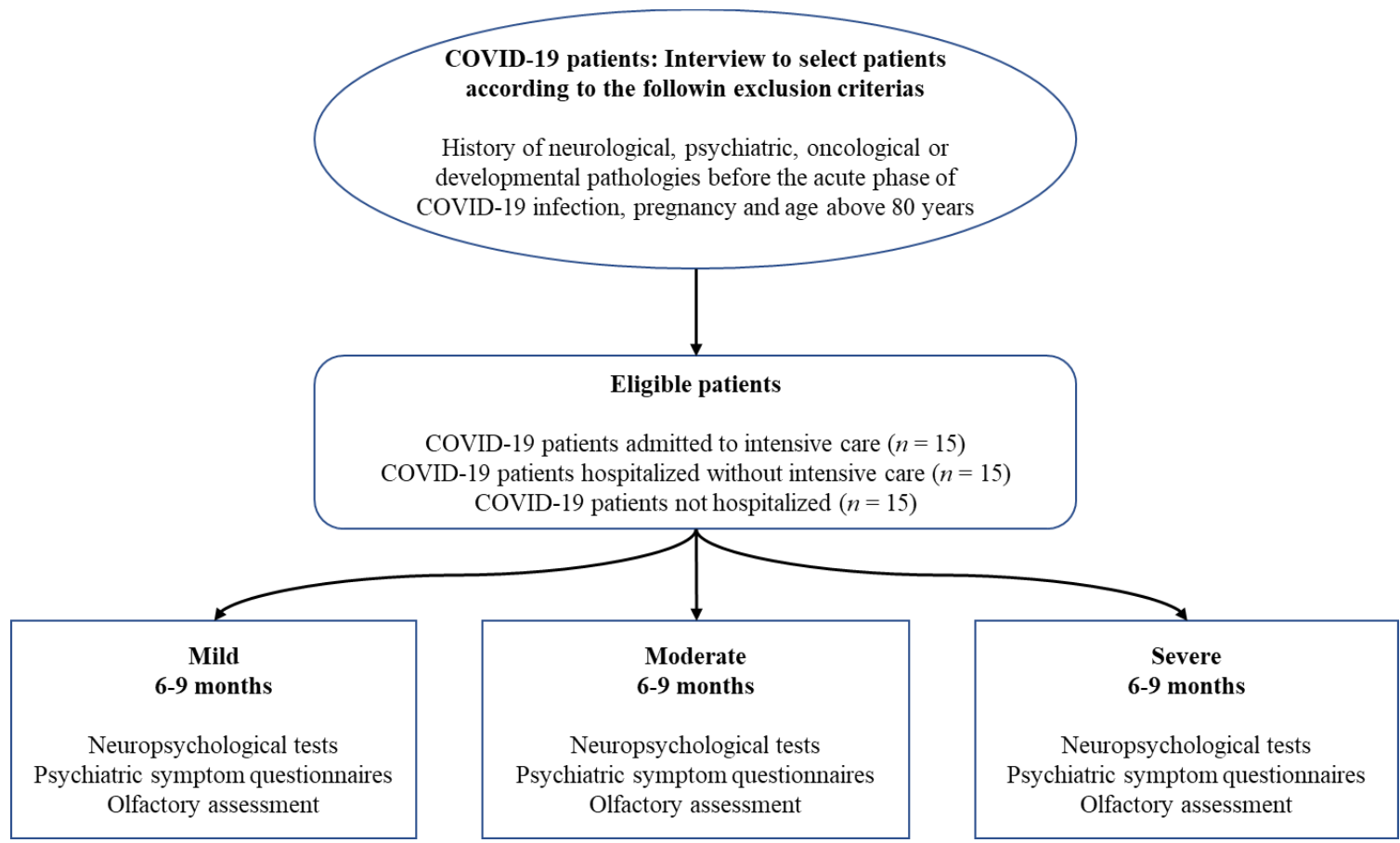

Figure 1: Flowchart of the study

\section{Neuropsychological assessment}

196 A comprehensive neuropsychological battery was administered to participants 6-9 months

197 after their positive PCR test $(236.51 \pm 22.54$ days $)$. This battery included a series of tests and

198 questionnaires assessing most of the domains of cognition, emotion recognition, fatigue, and

199 quality of life (see below). The tests were administered by clinical psychologists (mean 
medRxiv preprint doi: https://doi.org/10.1101/2021.02.24.21252329; this version posted February 26, 2021. The copyright holder for this preprint (which was not certified by peer review) is the author/funder, who has granted medRxiv a license to display the preprint in It is made available perpetuity.

Voruz et al. - Long COVID-19 and cognition

200 duration: approx. 180 minutes), and the questionnaires were administered online via Qualtrics 201 software (Qualtrics, Provo, UT) (mean duration: approx. 60 minutes).

Stroop task, Trail Making Test, and categorical and lexical verbal fluency from the GREFEX battery (Roussel \& Godefroy, 2008). Verbal and visuospatial working memory were assessed using the backward digit span (Drozdick, Raiford, Wahlstrom, \& Weiss, 2018) and backward

administered computer-based tasks designed to gauge focused attention, divided attention, Attentional Performance (TAP) (Zimmermann \& Fimm, 2007).

Memory systems. The short-term memory system was assessed with forward digit spans (Drozdick et al., 2018) and the Corsi test (Kessels et al., 2000). Verbal episodic memory was assessed with the 16-item Grober and Buschke free/cued recall (RL/RI 16)

214 paradigm (Grober \& Buschke, 1987), as it distinguishes between the cognitive subprocesses 215 of encoding, storage, and recall (Van der Linden et al., 2004). Visual episodic memory was 216 assessed with the delayed recall of the Rey-Osterrieth Complex Figure test (Meyers \& 217 Meyers, 1995). Instrumental function. Language was assessed with the BECLA battery (Macoir,

219 Gauthier, Jean, \& Potvin, 2016), ideomotor praxis with a short validated battery (Mahieux220 Laurent et al., 2009), visuoconstructive abilities with the Rey-Osterrieth Complex Figure test 221 (Meyers \& Meyers, 1995), and visuoperceptual functions with 4 subtests from the Visual Object and Space Perception battery (VOSP) (Warrington \& James, 1991) measuring object perception (fragmented letters, object decision) and spatial perception (localization of

224 numbers, analysis of cubes). 
medRxiv preprint doi: https://doi.org/10.1101/2021.02.24.21252329; this version posted February 26, 2021. The copyright holder for this preprint (which was not certified by peer review) is the author/funder, who has granted medRxiv a license to display the preprint in It is made available underpetuity.

Voruz et al. - Long COVID-19 and cognition

Logical reasoning. This was assessed using the Puzzle and Matrices subtests of the

Wechsler Adult Intelligence Scale-Fourth Edition (WAIS-4) (Wechsler, 2008).

Emotion. Multimodal emotion recognition was assessed with the Geneva Emotion

Recognition Test (GERT) (Schlegel, Grandjean, \& Scherer, 2014). In this emotion

recognition task, participants watched 42 video clips, in which 10 actors displayed 14

different emotions (pride, fun, joy, pleasure, relief, interest, anger, irritation, fear, anxiety, disgust, despair, sadness, surprise) while expressing nonverbal content. After each clip, participants were asked to choose one emotion from the list of 14 that best described the emotion played by the actor.

Anosognosia and cognitive complaints. We administered the Cognitive Complaints Questionnaire (QPC) (Thomas-Antérion, Ribas, Honoré-Masson, Million, \& Laurent, 2004) and the Behavior Rating Inventory of Executive Function-adult Version (BRIEF-A) (Roth, Gioia, \& Isquith, 2005). To quantify anosognosia, we calculated a self-appraisal discrepancy (SAD) score for each memory and executive domain evaluated by the QPC and BRIEF-A (Leicht, Berwig, \& Gertz, 2010; Rosen et al., 2010; Tondelli et al., 2018). First, we calculated standardized scores for the cognitive complaints, dividing the raw scores of the self-report questionnaires into four categories: $0=$ normal behavior; $1=$ limited influence on daily life; 2 $=$ noticeable influence on daily life; and 3 = substantial influence on daily life. Then, each standardized score yielded by one of these self-administered questionnaires of cognitive complaints was subtracted from the standardized score for the relevant function. For example, if a patient reported no memory disorders $(\mathrm{QPC}$ score $=3)$ but performed very poorly on Grober and Buschke (RL/RI 16) - delayed free recall (score $=0)$, he/she would exhibit anosognosia for memory dysfunction: 0 (standardized score on episodic memory test) -3 (score on self-questionnaire of memory complaints $)=-3$. SAD scores could therefore range from -3 to 3 , and any score below 0 indicated anosognosia. 
medRxiv preprint doi: https://doi.org/10.1101/2021.02.24.21252329; this version posted February 26, 2021. The copyright holder for this preprint (which was not certified by peer review) is the author/funder, who has granted medRxiv a license to display the preprint in perpetuity.

Voruz et al. - Long COVID-19 and cognition

\section{Other clinical outcomes}

251 We collected patients' sociodemographic data and medical history. Psychiatric data (including 252 those concerning current fatigue, insomnia, and somnolence), olfactory abilities, and quality of life at the time of the interview were also collected. Finally, a neurological assessment of CNS and peripheral nervous system functions and walking was carried out by two certified neurologists (FA and GA).

Sociodemographic and clinical data. In addition to age, collected during the inclusion interview, we recorded patients' gender, handedness, and education level. To complement information about previous neurological, psychiatric, and developmental conditions and cancer collected during the inclusion interview, we asked patients about previous cardiovascular disease, respiratory disorders, immunosuppression status, sleep apnea

syndrome, diabetes, and smoking. Participants were asked to describe the symptoms they had experienced both during the acute phase of infection and currently (6-9 months postinfection), and the number of days they had spent in hospital, where relevant. Psychiatric data. Depression was assessed with the Beck Depression InventorySecond edition (BDI-II) (Beck, Steer, \& Brown, 1996), anxiety with the State-Trait Anxiety Inventory (STAI-S and STAI-T) (Spielberger, Gorsuch, Lushene, Vagg, \& Jacobs, 1993), apathy and its distinct subtypes with the Apathy Motivation Index (AMI) (Ang, Lockwood, Apps, Muhammed, \& Husain, 2017), PTSD with the Posttraumatic Stress Disorder Checklist for DSM-5 (PCL-5) (Ashbaugh, Houle-Johnson, Herbert, El-Hage, \& Brunet, 2016), manic symptoms with the Goldberg Mania Inventory (Goldberg, 1993), dissociative symptoms in the patient's daily life with the Dissociative Experience Scale (DES) (Carlson \& Putnam, 1986), current stress perception with the Perceived Stress Scale - 14 items (PSS-14) (Lesage, Berjot, \& Deschamps, 2012), cognitive reappraisal of an emotional episode and expressive emotional suppression capacities with the Emotion Regulation Questionnaire (ERQ) (Gross \& 
medRxiv preprint doi: https://doi.org/10.1101/2021.02.24.21252329; this version posted February 26, 2021. The copyright holder for this preprint (which was not certified by peer review) is the author/funder, who has granted medRxiv a license to display the preprint in It is made available under a CC-BY-ND 4.0 International license .

Voruz et al. - Long COVID-19 and cognition

275 John, 2003) and susceptibility to others' emotions with the Emotion Contagion Scale (ECS)

276 (Doherty, 1997). Finally, fatigue was assessed with the French version of the Fatigue Impact

277 Scale (Debouverie, Pittion-Vouyovitch, Louis, \& Guillemin, 2007), potential sleeping

278 disorders with the Insomnia Severity Index (ISI) (Morin, 1993), and symptoms of sleepiness

279 in daily life with the Epworth Sleepiness Scale (Johns, 1991).

280 Olfaction. Olfactory performance was measured with the Sniffin' Sticks test battery.

281 This test consists of commercially available pens with 16 common odors, which were each

282 presented for $2 \mathrm{~s}$ in front of both nostrils. For each odor, patients had to choose between four

283 descriptors in a multiple-choice task. Participants' scores ranged from 0 to 16. Based on

284 Kobal et al. (2000), we set three thresholds. Patients with an identification score of 0-7 were

285 considered anosmic, 8-12 hyposmic, and 12-16 normosmic.

286 Quality of life. We administered the SF-36 (Bousquet et al., 1994), which distinguishes

287 between the physical and mental aspects of quality of life. 
medRxiv preprint doi: https://doi.org/10.1101/2021.02.24.21252329; this version posted February 26, 2021. The copyright holder for this preprint (which was not certified by peer review) is the author/funder, who has granted medRxiv a license to display the preprint in it is made available underpetuity.

Voruz et al. - Long COVID-19 and cognition

\section{Statistical analyses}

Prevalence of neuropsychological deficits and psychiatric symptoms (Objective 1)

291 For each neuropsychological test, we first compared patients' performances with normative

292 data for the validated neuropsychological tools. As the standardization depended upon the

293 distribution of the normative data collected from the reference sample ( $t$ and $z$ scores,

294 percentiles, or standard scores), the comparative tests were adjusted according to the guidelines provided by the authors of the validation study for each test. Second, the data were normalized according to the guidelines of the Swiss Association of Neuropsychology (Frei et al., 2016; Heaton, Grant, \& Matthews, 1991), making it possible to classify the standardized as follows: far below the norm $\left(<2^{\text {nd }}\right.$ percentile $)$, substandard $\left(2^{\text {nd }}-5^{\text {th }}\right.$ percentiles $)$, borderline or below the normal limit $\left(6^{\text {th }}-15^{\text {th }}\right.$ percentiles $)$, normal $\left(\geq 16^{\text {th }}\right.$ percentile $)$. This standardization allowed us to quantify the prevalence of each type of disorder, while controlling for variables such as age, education level, and gender. To consider the possible effect of fatigue and increase the robustness of the results, only those performances that were far below the norm $\left(<2^{\text {nd }}\right.$ percentile $)$ or substandard $\left(2^{\text {nd }}-5^{\text {th }}\right.$ percentile $)$ were used to calculate the prevalence of neuropsychological deficits. Results that were just below the norm were therefore not considered in the prevalence table.

308 For each neuropsychological, psychiatric or quality-of-life measure, we compared the three 309 groups (severe, moderate, and mild) on the raw data. Given the distribution of the samples, we 310 used nonparametric Kruskal-Wallis tests. For significant $(p<.05)$ measures, Mann-Whitney tests were performed for the $2 \times 2$ comparisons with Bonferroni-corrected $p$ values. 
medRxiv preprint doi: https://doi.org/10.1101/2021.02.24.21252329; this version posted February 26, 2021. The copyright holder for this preprint (which was not certified by peer review) is the author/funder, who has granted medRxiv a license to display the preprint in It is made available under a CC-BY-ND 4.0 International license .

Voruz et al. - Long COVID-19 and cognition secondary variables (Objective 3)

315 For each neuropsychological variable of interest, forward stepwise multiple regression

316 analyses were performed on the raw cognitive data with the significant sociodemographic

317 variables, sniff test results and psychiatric measures, to quantify relationships between these

318 variables and the neuropsychological functions.

319 In parallel, and in order to elucidate the cognitive data's underlying structure, we

320 performed a principal component analysis (PCA) on the raw test and questionnaire scores

321 assessing cognition and emotion recognition. The list of variables included in the PCA is

322 available in Supplementary Information 1. We extracted the first three components with the

323 highest eigenvalues. We then reran forward stepwise multiple regressions for each cognitive

324 component, with the same variables of interest as those described above. 
medRxiv preprint doi: https://doi.org/10.1101/2021.02.24.21252329; this version posted February 26, 2021. The copyright holder for this preprint (which was not certified by peer review) is the author/funder, who has granted medRxiv a license to display the preprint in

Voruz et al. - Long COVID-19 and cognition

\section{RESULTS}

\section{Neuropsychological, psychiatric and olfactory profiles 6-9 months post-}

\section{infection}

329 The first aim of this study was to assess the prevalence of neuropsychological impairments 330 and psychiatric symptoms 6-9 months after SARS-CoV-2 infection. We compared patients'

331 performances with available normative data to identify the number of impaired scores per

332 patient, group, and test. The prevalence of cognitive impairments in each group $236.51 \pm$

33322.54 days after infection is set out in Table 2, and the prevalence of psychiatric symptoms in

334 Table 3

Cognition. Cognitive deficits common to all three groups were observed in the

336 following domains: long-term episodic memory in both the verbal and visual modalities,

337 executive functions (e.g., inhibition and mental flexibility, and both categorical and literal

338 verbal fluency), sustained and divided attention, and language (semantic matching and

339 naming). All three groups exhibited anosognosia for executive dysfunction (see Table 2)

$340 \quad$ Psychiatric disorders. All three groups displayed anxiety, mania, the social component

341 of apathy, stress, PTSD, and dissociative disorders. All three groups also reported insomnia,

342 fatigue and pathological somnolence (see Table 3 ). The only psychiatric variable where the

343 prevalence score stood out for severe patients was emotional apathy, as measured with AMI

344 (see Table 3).

345 Olfaction. $33.33 \%$ of the mild group, $73.33 \%$ of the moderate group, and $46.66 \%$ of

346 the severe group displayed hyposmia. There was no anosmia in the mild and moderate groups,

347 but $13.33 \%$ of the severe group were anosmic (see Table 3 ). 
Table 2: Cognitive deficits among patients with mild, moderate or severe COVID-19 6-9 months post-infection

\begin{tabular}{|c|c|c|c|c|c|}
\hline & & Mild $(n=15)$ & Moderate $(n=15)$ & Severe $(n=15)$ & $p$ value \\
\hline & & Prevalence $(\%)$ & Prevalence $(\%)$ & Prevalence (\%) & \\
\hline \multicolumn{6}{|l|}{ Memory } \\
\hline \multirow[t]{5}{*}{ Verbal episodic memory } & Grober \& Buschke (RL/RI 16) - Immediate recall & 0.00 & 0.00 & 6.67 & .066 \\
\hline & Grober \& Buschke (RL/RI 16) - Sum of 3 free recalls & 0.00 & 0.00 & 0.00 & .110 \\
\hline & Grober \& Buschke (RL/RI 16) - Sum of 3 total recalls & 0.00 & 13.33 & 6.67 & .229 \\
\hline & Grober \& Buschke (RL/RI 16) - Delayed free recall & 0.00 & 6.67 & 6.67 & .005 \\
\hline & Grober \& Buschke (RL/RI 16) - Delayed total recall & 6.67 & 13.33 & 20.00 & .450 \\
\hline \multirow[t]{4}{*}{ Visuospatial episodic memory } & Rey Figure - Copy time & 0.00 & 0.00 & 0.00 & .459 \\
\hline & Rey Figure - Score & 6.67 & 20.00 & 6.67 & .904 \\
\hline & Rey Figure - Immediate recall (3') & 0.00 & 26.67 & 0.00 & .117 \\
\hline & Rey Figure - Delayed recall (20') & 0.00 & 13.33 & 0.00 & .046 \\
\hline Verbal short-term memory & MEM III -Spans & 0.00 & 6.67 & 6.67 & .351 \\
\hline Visuospatial short-term memory & WAIS IV - Spans & 0.00 & 0.00 & 0.00 & .424 \\
\hline \multicolumn{6}{|l|}{ Executive functions } \\
\hline Inhibition & Stroop (GREFEX) - Interference - Time & 26.67 & 0.00 & 13.33 & .112 \\
\hline
\end{tabular}




\begin{tabular}{|c|c|c|c|c|c|}
\hline & Stroop (GREFEX)- Interference - Errors & 6.67 & 28.57 & 13.33 & .312 \\
\hline & Stroop (GREFEX)- Interference/Naming - Score & 13.33 & 7.14 & 13.33 & .170 \\
\hline \multirow[t]{4}{*}{ Working memory } & MEM III - Verbal working memory & 6.67 & 0.00 & 0.00 & .273 \\
\hline & WAIS IV - Visuospatial working memory & 6.67 & 6.67 & 0.00 & .736 \\
\hline & TAP - Working memory item omissions & 0.00 & 0.00 & 7.14 & .664 \\
\hline & TAP - Working memory false alarms & 0.00 & 6.67 & 14.29 & .886 \\
\hline \multirow[t]{8}{*}{ Mental flexibility } & TMT A (GREFEX) - Time & 0.00 & 0.00 & 0.00 & .396 \\
\hline & TMT A (GREFEX) - Error & 0.00 & 6.67 & 13.33 & .343 \\
\hline & TMT B (GREFEX) - Time & 0.00 & 13.33 & 6.67 & .093 \\
\hline & TMT B (GREFEX) - Error & 6.67 & 13.33 & 20.00 & .543 \\
\hline & TMT B (GREFEX) - Perseverations & 13.33 & 33.33 & 20.00 & .140 \\
\hline & TMT B-A (GREFEX) - Score & 0.00 & 20.00 & 6.67 & .084 \\
\hline & Verbal fluency (GREFEX) - Literal (2') & 6.67 & 13.33 & 6.67 & .201 \\
\hline & Verbal fluency (GREFEX) - Categorical (2') & 13.33 & 20.00 & 26.67 & .246 \\
\hline \multirow[t]{4}{*}{ Incompatibility } & TAP - Compatibility - Reaction time & 0.00 & 0.00 & 0.00 & .834 \\
\hline & TAP - Compatibility - False alarms & 0.00 & 20.00 & 13.33 & .475 \\
\hline & TAP - Incompatibility - Reaction time & 0.00 & 0.00 & 0.00 & .548 \\
\hline & TAP - Incompatibility - False alarms & 0.00 & 20.00 & 13.33 & .528 \\
\hline
\end{tabular}




\begin{tabular}{|c|c|c|c|c|c|}
\hline Interhemispheric transfer & TAP - Incompatibility - Visual field score & 13.33 & 6.67 & 13.33 & .402 \\
\hline & TAP - Incompatibility task - Hands score & 6.67 & 6.67 & 0.00 & .631 \\
\hline \multicolumn{6}{|l|}{ Attentional functions } \\
\hline \multirow[t]{5}{*}{ Phasic alertness } & TAP - Without warning sound - Reaction time & 13.33 & 0.00 & 6.67 & .869 \\
\hline & TAP - Without warning sound - $S D$ of reaction time & 13.33 & 0.00 & 0.00 & .597 \\
\hline & TAP - With warning sound - Reaction time & 13.33 & 0.00 & 0.00 & .604 \\
\hline & TAP - With warning sound - $S D$ of reaction time & 6.67 & 0.00 & 0.00 & .256 \\
\hline & TAP - Alertness index & 6.67 & 6.67 & 0.00 & .426 \\
\hline \multirow[t]{2}{*}{ Sustained attention } & TAP - Item omissions & 13.33 & 30.77 & 7.69 & .657 \\
\hline & TAP - False alarm & 6.67 & 23.08 & 7.69 & .101 \\
\hline \multirow[t]{4}{*}{ Divided attention } & TAP - Audio condition - Reaction time & 20.00 & 13.33 & 13.33 & .242 \\
\hline & TAP - Visual condition - Reaction time & 6.67 & 0.00 & 0.00 & .999 \\
\hline & TAP - Total omissions & 6.67 & 13.33 & 0.00 & .748 \\
\hline & TAP - Total false alarms & 0.00 & 0.00 & 20.00 & .101 \\
\hline \multicolumn{6}{|l|}{ Instrumental functions } \\
\hline \multirow[t]{3}{*}{ Language } & BECLA - Semantic image matching & 6.67 & 6.67 & 13.33 & .409 \\
\hline & BECLA - Semantic word matching & 6.67 & 13.33 & 6.67 & .937 \\
\hline & BECLA - Object and action image naming & 6.67 & 6.67 & 6.67 & .879 \\
\hline
\end{tabular}


Voruz et al. - Long COVID-19 and cognition

\begin{tabular}{|c|c|c|c|c|c|}
\hline & BECLA - Word repetition & 0.00 & 0.00 & 0.00 & .369 \\
\hline & BECLA - Nonword repetition & 0.00 & 0.00 & 6.67 & .191 \\
\hline \multirow[t]{3}{*}{ Ideomotor praxis } & Evaluation of ideomotor praxis - Symbolic gestures & 0.00 & 0.00 & 0.00 & .057 \\
\hline & Evaluation of ideomotor praxis - Action pantomimes & 0.00 & 0.00 & 0.00 & .222 \\
\hline & Evaluation of ideomotor praxis - Meaningless gestures & 13.33 & 0.00 & 6.67 & .902 \\
\hline \multirow[t]{2}{*}{ Object perception } & VOSP - Fragmented letters & 0.00 & 6.67 & 0.00 & .690 \\
\hline & VOSP - Object decision & 20.00 & 33.33 & 0.00 & .229 \\
\hline \multirow[t]{2}{*}{ Spatial perception } & VOSP - Number localization & 0.00 & 20.00 & 6.67 & .932 \\
\hline & VOSP - Cubic counting & 0.00 & 6.67 & 0.00 & .772 \\
\hline \multirow[t]{2}{*}{ Logical reasoning } & WAIS IV - Puzzle & 6.67 & 6.67 & 0.00 & .299 \\
\hline & WAIS IV - Matrix & 0.00 & 13.33 & 0.00 & .223 \\
\hline \multirow[t]{4}{*}{ Anosognosia } & Memory functions & 0.00 & 40.00 & 40.00 & .020 \\
\hline & Executive functions - Inhibition & 20.00 & 26.67 & 53.33 & .508 \\
\hline & Executive functions - Flexibility & 20.00 & 33.33 & 33.33 & .503 \\
\hline & Executive functions - Working memory & 6.67 & 6.67 & 0.00 & .390 \\
\hline \multirow[t]{2}{*}{ Cognitive complaints } & $\overline{\mathrm{QPC}}$ & 6.67 & 13.33 & 0 & .041 \\
\hline & Executive complaints (BRIEF-5) (mean $\pm S D$ ) & $112.47( \pm 13.25)$ & $113.20( \pm 20.96)$ & $99.60( \pm 20.06)$ & .153 \\
\hline Emotion recognition & GERT - Emotion recognition task $($ mean $\pm S D)$ & $26.53( \pm 5.14)$ & $20.33( \pm 6.17)$ & $21.6( \pm 7.23)$ & .023 \\
\hline
\end{tabular}


Voruz et al. - Long COVID-19 and cognition

1 Note. Prevalence was calculated according to normative scores, ranging from much lower than the norm (below $2^{\text {nd }}$ percentile) to

2 substandard (between $2^{\text {nd }}$ and $5^{\text {th }}$ percentiles). Scores that were borderline or below the normal limit (between $6^{\text {th }}$ and $15^{\text {th }}$ percentiles) were not

3 included.

4 Abbreviations: BECLA: Batterie d'évaluation cognitive du langage (Macoir et al., 2016); GERT: Geneva Emotion Recognition Test

5 (Schlegel et al., 2014); GREFEX: Groupe de Réflexion sur l'Evalutation des Fonctions Exécutives (Roussel \& Godefroy, 2008); MEM III:

6 Wechsler Memory Scale - Third Edition (Drozdick et al., 2018); QPC: Cognitive Complaint Questionnaire (Thomas-Antérion et al., 2004); Rey

7 figure: Rey-Osterrieth Complex Figure test (Meyers \& Meyers, 1995); RL/RI-16: free/cued recall 16 items (Grober \& Buschke, 1987); SD:

8 standard deviation; TAP: Test for Attentional Performance (TAP), Version 2.1 (Zimmermann \& Fimm, 2007); TMT: Trail Making Test; VOSP:

9 Visual Object and Space Perception battery (Warrington \& James, 1991); WAIS IV: Wechsler Adult Intelligence Scale-Fourth Edition

10 (Wechsler, 2008). 
Voruz et al. - Long COVID-19 and cognition

\begin{tabular}{|c|c|c|c|c|}
\hline Psychiatric symptoms & Mild $(n=15)$ & Moderate $(n=15)$ & Severe $(n=15)$ & $p$ value \\
\hline $\begin{array}{l}\text { Depression (BDI-II) } \\
\text { (prevalence) }\end{array}$ & $\begin{array}{l}\text { Minor }=46.67 \% \\
\text { Mild }=20 \% \\
\text { Moderate }=33.33 \% \\
\text { Severe }=0 \%\end{array}$ & $\begin{array}{l}\text { Minor }=66.67 \% \\
\text { Mild }=20 \% \\
\text { Moderate }=13.33 \% \\
\text { Severe }=0 \%\end{array}$ & $\begin{array}{l}\text { Minor }=80 \% \\
\text { Mild }=20 \% \\
\text { Moderate }=0 \% \\
\text { Severe }=0 \%\end{array}$ & .009 \\
\hline $\begin{array}{l}\text { State anxiety (STAI-state) } \\
\text { (prevalence) }\end{array}$ & $\begin{array}{l}\text { Very low }=26.67 \% \\
\text { Low }=33.33 \% \\
\text { Moderate }=13.33 \% \\
\text { High }=26.67 \% \\
\text { Very high }=0 \%\end{array}$ & $\begin{array}{l}\text { Very low }=60 \% \\
\text { Low }=6.67 \% \\
\text { Moderate }=20 \% \\
\text { High }=13.33 \% \\
\text { Very high }=0 \%\end{array}$ & $\begin{array}{l}\text { Very low }=86.67 \% \\
\text { Low }=6.67 \% \\
\text { Moderate }=6.67 \% \\
\text { High }=0 \% \\
\text { Very high }=0 \%\end{array}$ & .002 \\
\hline $\begin{array}{l}\text { Trait anxiety (STAI-trait) } \\
\text { (prevalence) }\end{array}$ & $\begin{array}{l}\text { Very low }=46.67 \% \\
\text { Low }=26.67 \% \\
\text { Moderate }=13.33 \% \\
\text { High }=13.33 \% \\
\text { Very high }=0 \%\end{array}$ & $\begin{array}{l}\text { Very low }=73.33 \% \\
\text { Low }=0 \% \\
\text { Moderate }=20 \% \\
\text { High }=6.67 \% \\
\text { Very high }=0 \%\end{array}$ & $\begin{array}{l}\text { Very low }=60 \% \\
\text { Low }=33.33 \% \\
\text { Moderate }=6.67 \% \\
\text { High }=0 \% \\
\text { Very high }=0 \%\end{array}$ & .100 \\
\hline
\end{tabular}




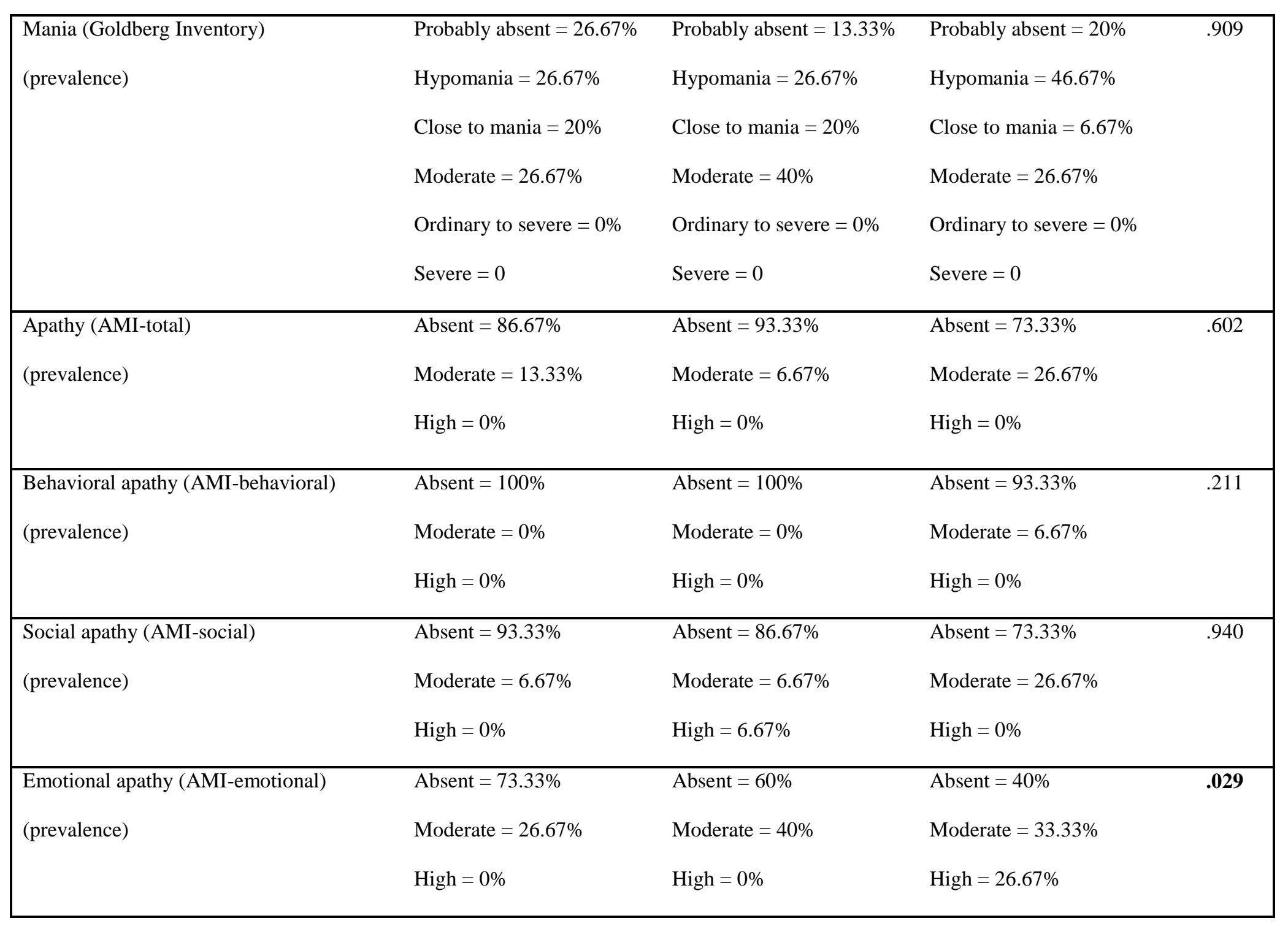


Voruz et al. - Long COVID-19 and cognition

\begin{tabular}{|c|c|c|c|c|}
\hline $\begin{array}{l}\text { Posttraumatic stress disorder (PCL-5) } \\
\text { (prevalence) }\end{array}$ & $\begin{array}{l}\text { Absent }=86.67 \% \\
\text { Present }=13.33 \%\end{array}$ & $\begin{array}{l}\text { Absent }=86.67 \% \\
\text { Present }=13.33 \%\end{array}$ & $\begin{array}{l}\text { Absent }=93.33 \% \\
\text { Present }=6.67 \%\end{array}$ & .054 \\
\hline $\begin{array}{l}\text { Stress (PSS-14) } \\
\text { Mean }( \pm S D)\end{array}$ & $26.13( \pm 9.53)$ & $19.6( \pm 7.47)$ & $14.93( \pm 9.42)$ & .023 \\
\hline $\begin{array}{l}\text { Dissociative disorder (DES) } \\
\text { Mean }( \pm S D)\end{array}$ & $7.68( \pm 11.89)$ & $10.45( \pm 9.23)$ & $3.98( \pm 3.03)$ & .140 \\
\hline $\begin{array}{l}\text { Emotional contagion (ECS) } \\
\text { Mean }( \pm S D)\end{array}$ & $41.40( \pm 7.20)$ & $44.6( \pm 4.22)$ & $36.13( \pm 8.38)$ & .002 \\
\hline $\begin{array}{l}\text { Emotional regulation (ERQ) } \\
\text { Mean }( \pm S D)\end{array}$ & $41.6( \pm 7.39)$ & $44.2( \pm 8.33)$ & $39.80( \pm 11.77)$ & .416 \\
\hline $\begin{array}{l}\text { Somnolence (Epworth) } \\
\text { (prevalence) }\end{array}$ & Pathological $=40.00 \%$ & Pathological $=53.33 \%$ & Pathological $=26.67 \%$ & .036 \\
\hline $\begin{array}{l}\text { Insomnia (ISI) } \\
\text { (prevalence) }\end{array}$ & $\begin{array}{l}\text { Absent }=20 \% \\
\text { Mild }=40 \% \\
\text { Moderate }=40 \% \\
\text { Severe }=0 \%\end{array}$ & $\begin{array}{l}\text { Absent }=46.67 \% \\
\text { Mild }=40 \% \\
\text { Moderate }=13.33 \% \\
\text { Severe }=0 \%\end{array}$ & $\begin{array}{l}\text { Absent }=60 \% \\
\text { Mild }=26.67 \% \\
\text { Moderate }=13.33 \% \\
\text { Severe }=0 \%\end{array}$ & .040 \\
\hline
\end{tabular}




\begin{tabular}{|llll|}
\hline Fatigue $($ EMIF-SEP) & Present $=13.33 \%$ & Present $=20 \%$ & Present $=6.67 \%$ \\
(prevalence) & & & \\
& & & \\
\hline Sniff test (anosmia) & $13.07( \pm 1.44)$ & $11.53( \pm 2.13)$ & $11.47( \pm 2.90)$ \\
Mean $( \pm S D)$ & & & .067 \\
& & & \\
\end{tabular}

Abbreviations: AMI-behavioral: Apathy Motivation Index - behavioral score (Ang et al., 2017); AMI-emotional: Apathy Motivation

14 Index - emotional score (Ang et al., 2017); AMI-social: Apathy Motivation Index - social score (Ang et al., 2017); AMI-total: Apathy

15 Motivation Index - total score (Ang et al., 2017); BDI-II: Beck Depression Inventory-Second edition (Beck et al., 1996); DES: Dissociative

16 Experience Scale (Carlson \& Putnam, 1986); ECS: Emotion Contagion Scale (Doherty, 1997); EMIF-SEP: Fatigue Impact Scale, French

17 adaptation (Debouverie et al., 2007); ERQ: Emotion Regulation Questionnaire (Gross \& John, 2003); Epworth: Epworth Sleepiness Scale (Johns,

18 1991); Goldberg Inventory: Goldberg Mania Inventory (Goldberg, 1993); ISI: Insomnia Severity Index (Morin, 1993); PCL-5: Posttraumatic

19 Stress Disorder Checklist for DSM-5 (Ashbaugh et al., 2016); PSS-14: Perceived Stress Scale - 14 items (Lesage et al., 2012); STAI-trait: State-

20 Trait Anxiety Inventory (Spielberger et al., 1993); STAI-state: State-Trait Anxiety Inventory (Spielberger et al., 1993). 


\section{Neuropsychological and psychiatric symptoms as a function of disease}

\section{severity}

The second aim was to determine whether cognitive deficits and psychiatric symptoms are a function of the severity of the respiratory symptoms in the acute phase. To this end, we compared the three groups on neuropsychological, psychiatric and other clinical data (Kruskal-Wallis statistics and $p$ values reported in Tables 2, 3 and 4; Bonferroni-corrected Mann-Whitney statistics and $p$ values reported below).

\section{i) Neuropsychological data (Fig. 2)}

The three groups differed significantly on i) long-term episodic memory in both the verbal (Grober and Buschke (RL/RI 16) delayed free recall, $H=10.75, p=.005)$ and visual (Rey Figure delayed free recall, $H=6.15, p=.046$ ) modalities, ii) multimodal emotion recognition (GERT; $H=7.55, p=.023)$, iii) cognitive complaints $(\mathrm{QPC} ; H=6.38, p=.041)$ and anosognosia for memory dysfunction (SAD; $H=7.84, p=.020)$. The other effects were not significant $(p>.05$ for all comparisons).

Episodic memory. For Grober and Buschke delayed free recall, the mild patients scored significantly higher than the severe patients $(z=3.04, p=.002)$, but the other two pairwise comparisons were not significant after Bonferroni correction (moderate vs. severe: $z$ $=-1.47, p=.141$; mild vs. moderate: $z=2.00, p=.046$ ). Pairwise comparisons were not significant for visual episodic memory (mild vs. moderate: $z=2.26, p=.023$; mild vs. severe: $z=0.48, p=.61 ;$ moderate vs. severe: $z=1.89, p=.059)$.

Emotion recognition. Mild patients scored significantly higher than moderate patients $(z=2.61, p=0.009)$, but neither the difference between mild and severe patients $(z=1.97, p$ $=0.048)$, nor the difference between moderate and severe patients $(z=.49, p=.620)$ reached significance after Bonferroni correction. 
medRxiv preprint doi: https://doi.org/10.1101/2021.02.24.21252329; this version posted February 26, 2021. The copyright holder for this preprint (which was not certified by peer review) is the author/funder, who has granted medRxiv a license to display the preprint in perpetuity.

Long COVID-19 and cognition

than severe patients $(z=-2.55, p=.010)$, but there were no differences between either the mild and moderate patients $(z=-1.31, p=.191)$, or the moderate and severe patients $(z=-$ patients $(z=-0.76, p=.443)$.
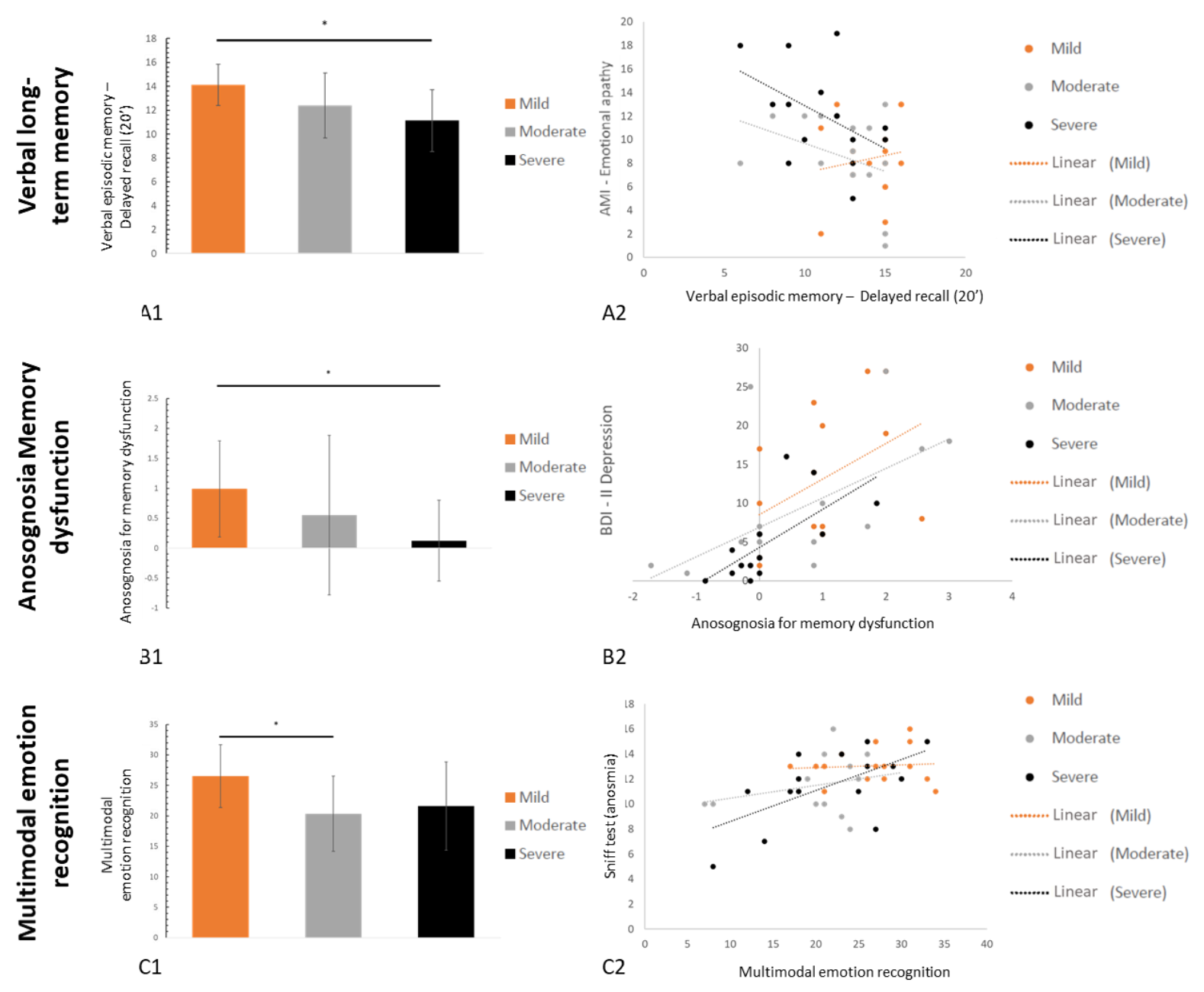

Figure 2. Mean ratings (and standard deviations) for all three groups (severe in 
medRxiv preprint doi: https://doi.org/10.1101/2021.02.24.21252329; this version posted February 26, 2021. The copyright holder for this preprint (which was not certified by peer review) is the author/funder, who has granted medRxiv a license to display the preprint in

Long COVID-19 and cognition

(A1), anosognosia for memory dysfunction (B1), and multimodal emotion recognition

(C1), as well as their respective predictors (A2, B2 and $\mathrm{C} 2)$

Note. A2: The greater the emotional apathy, the poorer the performance on verbal emotion recognition. anxiety $(H=12.93, p=.002)$, emotional apathy $(H=7.10, p=.029)$, stress $(H=7.55, p=$ $.023)$, and emotional contagion $(H=9.73, p=.002)$. The other effects were not significant ( $p$ $>.05$ for all comparisons). Pairwise comparisons for each of these group differences are described below.

Depression, stress, and state anxiety. The mild patients were more depressed, stressed and anxious than the severe patients (BDI-II: $z=-2.99, p=.003$; PSS: $z=-2.55, p=.010$; STAI-S: $z=-3.57, p<.001$ ), while there were no differences between either the severe and moderate patients (BDI-II: $z=-1.38, p=.165$; PSS: $z=-1.08, p=.281$; STAI-S: $z=-1.76, p$

$73=.078$ ) or the mild and moderate patients (BDI II: $z=-1.66, p=.097$; PSS: $z=-1.08, p=$ .281 ; STAI-S: $z=-1.72, p=.085)$. significance after Bonferroni correction (severe vs. mild: $z=2.32, p=.020$; severe vs. moderate: $z=2.20, p=.028$, mild vs. moderate: $z=0.08, p=.933$ ). 
medRxiv preprint doi: https://doi.org/10.1101/2021.02.24.21252329; this version posted February 26, 2021. The copyright holder for this preprint (which was not certified by peer review) is the author/funder, who has granted medRxiv a license to display the preprint in

Long COVID-19 and cognition

Emotional contagion. Pairwise comparisons failed to reach significance after

\section{iii) Fatigue and quality of life}

Finally, the three groups differed on insomnia $(H=6.66, p=.036)$, fatigue $(H=6.45$, $p=.040)$, vitality $(H=6.50, p=.039)$, and emotional wellbeing $(H=9.18, p=.010)$. The other effects were not significant ( $p>.05$ for all comparisons).

The mild patients reported more fatigue than the severe patients $(z=-2.57, p=.010)$, while there were no differences between either the mild and moderate patients $(z=-0.71, p=$ $.481)$ or both moderate and severe patients $(z=-1.52, p=.130)$. Pairwise comparisons did not reach significance after Bonferroni correction (mild vs. moderate: $z=-1.99, p=.046$; mild vs. severe: $z=-2.28, p=.023$; moderate vs. severe: $z=-0.71, p=.481)$.

Conversely, severe patients reported more vitality, emotional wellbeing and social function than mild patients (vitality: $z=2.65, p=.008$; wellbeing: $z=2.97, p=.003$; social function: $z=2.99, p=.002$ ). Pairwise comparisons between severe and moderate patients did not reach significance after Bonferroni correction (wellbeing: $z=2.01, p=.044$; vitality: $z=$ 1.06, $p=.290$; social function: $z=1.66, p=.097$ ), nor did those between mild and moderate patients (wellbeing: $z=0.68, p=.494$; vitality: $z=1.12, p=.263$; social function: $z=1.06, p$ $=.290)($ see Table 4). 
medRxiv preprint doi: https://doi.org/10.1101/2021.02.24.21252329; this version posted February 26, 2021. The copyright holder for this preprint (which was not certified by peer review) is the author/funder, who has granted medRxiv a license to display the preprint in It is made available under a CC-BY-ND 4.0 International license.

Long COVID-19 and cognition

101

\begin{tabular}{|lcccc|}
\hline Quality of life domains (SF-36) $^{+}$ & Mild $(n=15)$ & Moderate $(n=15)$ & Severe $(n=15)$ & $\boldsymbol{p}$ value \\
& Mean $( \pm \mathbf{S D})$ & Mean $( \pm \mathbf{S D})$ & Mean $( \pm \mathbf{S D})$ & \\
\hline Overall health & $62.67( \pm 16.89)$ & $59.33( \pm 27.31)$ & $66.00( \pm 24.14)$ & .808 \\
\hline Physical function & $80.00( \pm 17.22)$ & $82.33( \pm 19.44)$ & $77.33( \pm 24.41)$ & .806 \\
\hline Physical role & $58.33( \pm 30.86)$ & $53.33( \pm 43.16)$ & $71.67( \pm 36.43)$ & .353 \\
\hline Emotional role & $64.45( \pm 36.67)$ & $73.34( \pm 36.08)$ & $80.00( \pm 37.38)$ & .314 \\
\hline Social function & $57.50( \pm 23.05)$ & $66.67( \pm 31.93)$ & $85.00( \pm 18.42)$ & $\mathbf{. 0 1 1}$ \\
\hline Physical pain & $57.83( \pm 20.81)$ & $72.00( \pm 29.40)$ & $71.83( \pm 25.61)$ & .153 \\
\hline Emotional wellbeing & $58.13( \pm 17.75)$ & $61.33( \pm 24.96)$ & $79.2( \pm 17.90)$ & $\mathbf{. 0 1 0}$ \\
\hline Vitality score & $38.66( \pm 16.20)$ & $49.00( \pm 27.14)$ & $56.00( \pm 14.17)$ & $\mathbf{0 3 9}$ \\
\hline Health modification & $30.00( \pm 16.90)$ & $35.00( \pm 24.64)$ & $43.33( \pm 17.59)$ & .143 \\
\hline
\end{tabular}

102

$103+$ The higher the score, the better the quality of life. 
medRxiv preprint doi: https://doi.org/10.1101/2021.02.24.21252329; this version posted February $26,2021$. The copyright holder for this preprint (which was not certified by peer review) is the author/funder, who has granted medRxiv a license to display the preprint in

Long COVID-19 and cognition

105 Relationships between neuropsychological deficits, psychiatric symptoms,

106 and other secondary variables

107 The third aim was to examine whether the presence of long-term neuropsychological deficits

108 was correlated with psychiatric symptoms and/or other clinically relevant variables.

109 The results of the multiple regression performed on each cognitive variable are set out

110 in Table 5. Interestingly, apathy, depression, anxiety, emotion regulation, emotion contagion,

111 stress, PTSD, dissociative disorders, anosmia and diabetes all proved to be variables of

112 interest when it came to explaining the neuropsychological sequelae. Therefore, both

113 psychiatric and nonpsychiatric data correlated with neuropsychological deficits across the

114 three groups. There were at least three patterns of results, depending on the

115 neuropsychological domain: i) patterns in which neuropsychological sequelae did not

116 correlate with any psychiatric variables, but did with other clinical variables, such as

117 visuospatial long-term episodic memory (delayed recall of Rey-Osterrieth complex figure); ii)

118 patterns in which neuropsychological sequelae correlated with both psychiatric and clinical

119 variables, such as the object and action naming task scores (language); and iii) patterns in

120 which neuropsychological sequelae only correlated with psychiatric variables, such as

121 categorical verbal fluency. There was also a fourth possible pattern where the

122 neuropsychological sequelae correlated neither with psychiatric variables nor with clinical

123 ones, such as the score on the object decision task (object perception).

124 To reduce the dimensionality of the dataset, we computed a PCA. We selected the first

125 three orthogonal components accounting for $43.67 \%$ of the total variance. The first

126 component, accounting for $26.75 \%$ of the total variance, was difficult to interpret in terms of

127 underlying cognitive processes, as it included language (semantic word and image matching),

128 executive functions (mental flexibility), verbal episodic memory, and emotion recognition. 
medRxiv preprint doi: https://doi.org/10.1101/2021.02.24.21252329; this version posted February 26, 2021. The copyright holder for this preprint (which was not certified by peer review) is the author/funder, who has granted medRxiv a license to display the preprint in It is made available under a CC-BY-ND 4.0 International license .

Long COVID-19 and cognition

129 Interestingly, these happened to be precisely the variables on which the three groups differed

130 significantly (see Section "Neuropsychological and psychiatric symptoms as a function of

131 disease severity"). We therefore labeled this component respiratory disease severity. The

132 second component (9.79\% of total variance) was labeled attention and anosognosia, as it

133 included alertness, divided attention, and anosognosia for executive dysfunction. The third

134 component (7.15\% of total variance) was labeled instrumental functions, as it included

135 language, visual perception, and ideomotor praxis.

$136 \quad$ For the respiratory disease severity component, the best fit was achieved with

137 emotional apathy $\left(R^{2}=.28, p=.007\right)$, stress $\left(R^{2}=.19, p=.013\right)$, and anosmia $\left(R^{2}=.11, p=\right.$

138 .03). For the attention and anosognosia component, the multiple regression was not significant

$139(p>.1)$. For the instrumental functions component, the best fit was achieved with anosmia $\left(R^{2}\right.$

$140=.23, p=.04)$, mania $\left(R^{2}=.23, p=.006\right)$, and social apathy $\left(R^{2}=.17, p=.004\right)$. 
medRxiv preprint doi: https://doi.org/10.1101/2021.02.24.21252329; this version posted February 26, 2021. The copyright holder for this preprint (which was not certified by peer review) is the author/funder, who has granted medRxiv a license to display the preprint in perpetuity

Long COVID-19 and cognition

142 Table 5. Multiple regression results for each of the neuropsychological variables

Regressor

$R^{2} \quad p$ value

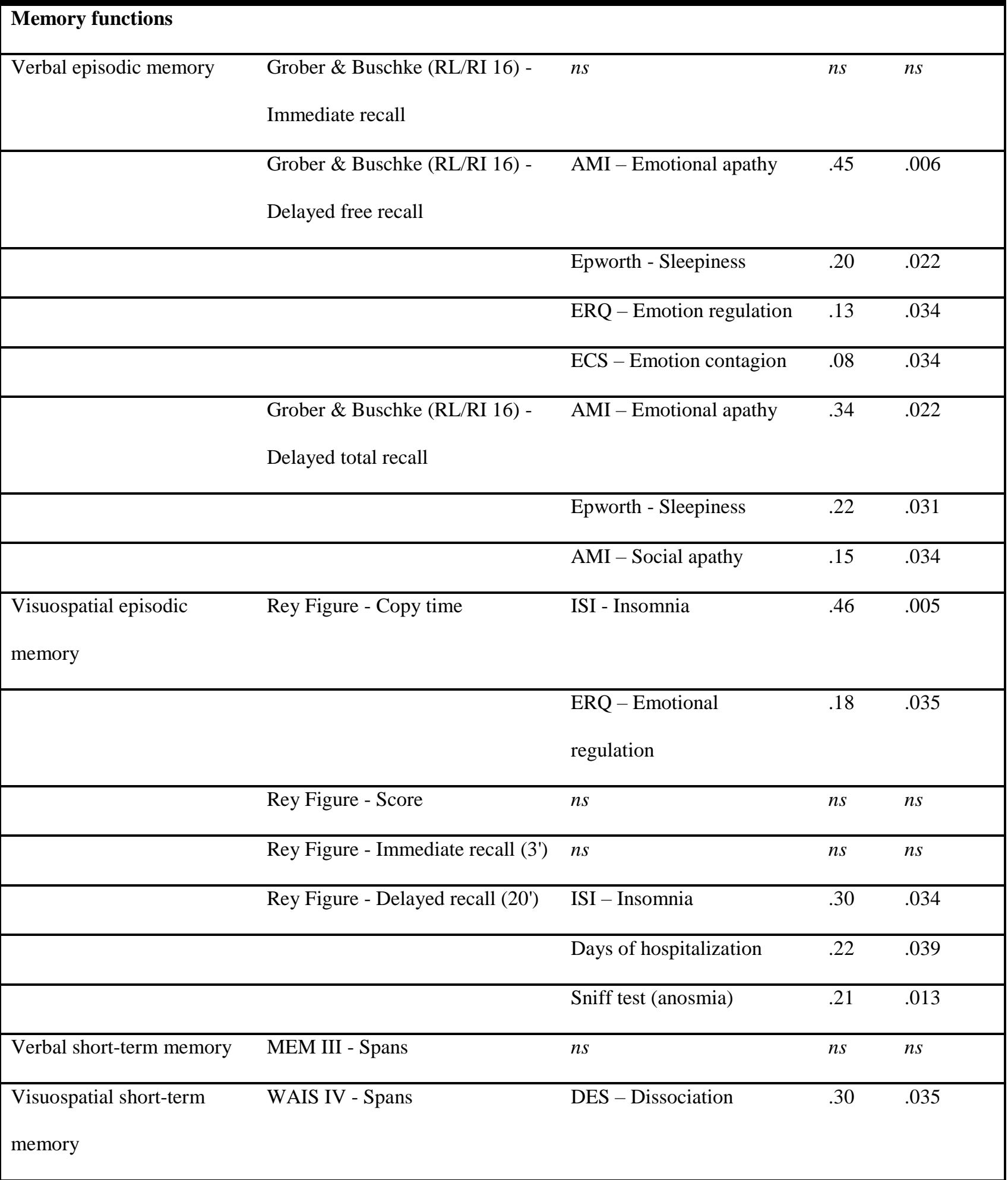


medRxiv preprint doi: https://doi.org/10.1101/2021.02.24.21252329; this version posted February 26, 2021. The copyright holder for this preprint (which was not certified by peer review) is the author/funder, who has granted medRxiv a license to display the preprint in It is made avaitabetuity.

perpetuity.
a CC-BY-ND 4.0 International license.

Long COVID-19 and cognition

\begin{tabular}{|c|c|c|c|c|}
\hline Executive functions & & & & \\
\hline \multirow[t]{4}{*}{ Inhibition } & $\begin{array}{l}\text { Stroop (GREFEX) - Interference } \\
\text { - Time }\end{array}$ & $n s$ & $n s$ & $n s$ \\
\hline & $\begin{array}{l}\text { Stroop (GREFEX)- Interference - } \\
\text { Errors }\end{array}$ & AMI - Total apathy & .37 & .015 \\
\hline & & ERQ - Emotion regulation & .21 & .030 \\
\hline & $\begin{array}{l}\text { Stroop (GREFEX) - } \\
\text { Interference/Naming - Score }\end{array}$ & $n s$ & $n s$ & $n s$ \\
\hline \multirow[t]{7}{*}{ Working memory } & $\begin{array}{l}\text { MEM III - Verbal working } \\
\text { memory }\end{array}$ & AMI - Behavioral apathy & .33 & .026 \\
\hline & $\begin{array}{l}\text { WAIS IV - Visuospatial working } \\
\text { memory }\end{array}$ & STAI-T Anxiety & .39 & .013 \\
\hline & & Diabetes & .25 & .014 \\
\hline & & STAI-S Anxiety & .13 & .03 \\
\hline & $\begin{array}{l}\text { TAP - Working memory item } \\
\text { omissions }\end{array}$ & AMI - Emotional apathy & .30 & .035 \\
\hline & $\begin{array}{l}\text { TAP - Working memory false } \\
\text { alarms }\end{array}$ & Diabetes & .47 & .005 \\
\hline & & $\begin{array}{l}\text { Mania-Goldberg } \\
\text { Inventory }\end{array}$ & .16 & .044 \\
\hline \multirow[t]{6}{*}{ Mental flexibility } & TMT A (GREFEX) - Time & $n s$ & $n s$ & $n s$ \\
\hline & TMT A (GREFEX) - Errors & $n s$ & $n s$ & $n s$ \\
\hline & TMT B (GREFEX) - Time & $n s$ & $n s$ & $n s$ \\
\hline & TMT B (GREFEX) - Errors & AMI - Total apathy & .41 & .010 \\
\hline & & ERQ - Emotion regulation & .14 & .024 \\
\hline & & Gender & .13 & .025 \\
\hline
\end{tabular}


medRxiv preprint doi: https://doi.org/10.1101/2021.02.24.21252329; this version posted February 26, 2021. The copyright holder for this preprint (which was not certified by peer review) is the author/funder, who has granted medRxiv a license to display the preprint in It is made available perpetuity.

perpetuity.
a CC-BY-ND 4.0 International license.

Long COVID-19 and cognition

\begin{tabular}{|c|c|c|c|c|}
\hline & $\begin{array}{l}\text { TMT B (GREFEX) - } \\
\text { Perseverations }\end{array}$ & STAI-T Anxiety & .55 & .002 \\
\hline & & BDI-II - Depression & .16 & .026 \\
\hline & & ISI - Insomnia & .20 & $<.001$ \\
\hline & TMT B-A (GREFEX) - Score & $n s$ & $n s$ & $n s$ \\
\hline & $\begin{array}{l}\text { Verbal fluency (GREFEX) - } \\
\text { Literal (2') }\end{array}$ & $n s$ & $n s$ & $n s$ \\
\hline & $\begin{array}{l}\text { Verbal fluency (GREFEX) - } \\
\text { Categorical fluency }\left(2^{\prime}\right)\end{array}$ & DES - Dissociation & .28 & .047 \\
\hline \multirow[t]{12}{*}{ Incompatibility } & $\begin{array}{l}\text { TAP - Compatibility - Reaction } \\
\text { time }\end{array}$ & AMI - Social apathy & .50 & .003 \\
\hline & $\begin{array}{l}\text { TAP - Compatibility - False } \\
\text { alarms }\end{array}$ & $n s$ & $n s$ & $n s$ \\
\hline & $\begin{array}{l}\text { TAP - Incompatibility - Reaction } \\
\text { Time }\end{array}$ & Sniff test (anosmia) & .28 & .043 \\
\hline & & DES - Dissociation & .25 & .026 \\
\hline & & ERQ - Emotion regulation & .13 & .028 \\
\hline & & Epworth - Sleepiness & .10 & .017 \\
\hline & $\begin{array}{l}\text { TAP - Incompatibility - False } \\
\text { alarms }\end{array}$ & Sniff test (anosmia) & .27 & .045 \\
\hline & $\begin{array}{l}\text { TAP - Incompatibility - Visual } \\
\text { field score }\end{array}$ & Days of hospitalization & .39 & .013 \\
\hline & & Diabetes & .23 & .007 \\
\hline & & STAI-T Anxiety & .08 & .041 \\
\hline & & $\begin{array}{l}\text { PCL-5 Posttraumatic stress } \\
\text { disorder }\end{array}$ & .06 & .041 \\
\hline & $\begin{array}{l}\text { TAP - Incompatibility task - } \\
\text { Hands score }\end{array}$ & AMI - Social apathy & .44 & .008 \\
\hline
\end{tabular}


medRxiv preprint doi: https://doi.org/10.1101/2021.02.24.21252329; this version posted February 26, 2021. The copyright holder for this preprint (which was not certified by peer review) is the author/funder, who has granted medRxiv a license to display the preprint in It is made available underty.

perpetuity.

Long COVID-19 and cognition

\begin{tabular}{|c|c|c|c|c|}
\hline & $\begin{array}{l}\text { TAP - Incompatibility task - } \\
\text { Visual fields * Hands score }\end{array}$ & ISI - Insomnia & .33 & .025 \\
\hline & & STAI-T Anxiety & .24 & .025 \\
\hline & & BDI-II - Depression & .18 & .019 \\
\hline \multicolumn{5}{|c|}{ Attentional functions } \\
\hline \multirow[t]{7}{*}{ Phasic alertness } & $\begin{array}{l}\text { TAP - Without warning sound - } \\
\text { Reaction time }\end{array}$ & Gender & .35 & .019 \\
\hline & $\begin{array}{l}\text { TAP - Without warning sound - } \\
S D \text { of reaction time }\end{array}$ & AMI - Social apathy & .64 & $<.001$ \\
\hline & & Diabetes & .11 & .041 \\
\hline & & Sniff test (anosmia) & .10 & .023 \\
\hline & $\begin{array}{l}\text { TAP - With warning sound - } \\
\text { Reaction time }\end{array}$ & DES - Dissociation & .30 & .033 \\
\hline & $\begin{array}{l}\text { TAP - With warning sound - } S D \\
\text { of reaction time }\end{array}$ & $\overline{n s}$ & $n s$ & $n s$ \\
\hline & TAP - Alertness index & Gender & .28 & .041 \\
\hline \multirow[t]{2}{*}{ Sustained attention } & TAP - Items Omissions & STAI-Trait Anxiety & .46 & .005 \\
\hline & TAP - False alarm & $n s$ & $n s$ & $n s$ \\
\hline \multirow[t]{8}{*}{ Divided attention } & $\begin{array}{l}\text { TAP - Audio condition - Reaction } \\
\text { time }\end{array}$ & DES - Dissociation & .41 & .010 \\
\hline & & AMI - Behavioral apathy & .27 & .008 \\
\hline & $\begin{array}{l}\text { TAP - Visual condition - } \\
\text { Reaction time }\end{array}$ & Days of hospitalization & .38 & .014 \\
\hline & & Sniff test (anosmia) & .27 & .009 \\
\hline & & ISI - Insomnia & .23 & $<.001$ \\
\hline & & AMI - Emotional apathy & .05 & .016 \\
\hline & TAP - Total omissions & $n s$ & $n s$ & $n s$ \\
\hline & TAP - Total false alarms & AMI - Emotional apathy & .32 & .029 \\
\hline
\end{tabular}


medRxiv preprint doi: https://doi.org/10.1101/2021.02.24.21252329; this version posted February 26, 2021. The copyright holder for this preprint (which was not certified by peer review) is the author/funder, who has granted medRxiv a license to display the preprint in

Long COVID-19 and cognition

\subsection{International license.}

\begin{tabular}{lll} 
Epworth - Sleepiness & .28 & .015 \\
\hline AMI - Social apathy & .16 & .023
\end{tabular}

\section{Instrumental functions}

\begin{tabular}{|lll}
\hline Language & BECLA - Semantic image & $n s$ \\
& matching & \\
\hline & BECLA - Semantic word & $n s$ \\
& matching & \\
\hline & BECLA - Object and action & ECS - Emotional contagion \\
.38 &
\end{tabular}

\begin{tabular}{|c|c|c|c|c|}
\hline & & STAI-State Anxiety & .29 & .007 \\
\hline & & AMI - Emotional apathy & .10 & .014 \\
\hline & & ISI - Insomnia & .08 & .012 \\
\hline & BECLA - Word repetition & NV & NV & $\mathrm{NV}$ \\
\hline & BECLA - Nonword repetition & $n s$ & $n s$ & $n s$ \\
\hline \multirow[t]{5}{*}{ Ideomotor praxis } & Symbolic gestures & ERQ - Emotion regulation & .35 & .019 \\
\hline & & AMI - Behavioral apathy & .33 & .004 \\
\hline & Action pantomimes & BDI-II - Depression & .51 & .003 \\
\hline & Meaningless gestures & AMI - Total apathy & .30 & .033 \\
\hline & & AMI - Social apathy & .18 & .035 \\
\hline \multirow[t]{2}{*}{ Object perception } & VOSP - Fragmented letters & AMI - Total apathy & .26 & .029 \\
\hline & VOSP - Object decision & $n s$ & $n s$ & $n s$ \\
\hline \multirow[t]{4}{*}{ Spatial perception } & VOSP - Number localization & $n s$ & $n s$ & $n s$ \\
\hline & VOSP - Cubic counting & Mania-Goldberg & .27 & .047 \\
\hline & & Inventory & & \\
\hline & & PSS - Stress & .24 & .034 \\
\hline \multirow[t]{3}{*}{ Logical reasoning } & WAIS IV - Puzzle & Diabetes & .34 & .021 \\
\hline & WAIS IV - Matrix & DES - Dissociation & .28 & .041 \\
\hline & & Gender & .40 & .002 \\
\hline
\end{tabular}


medRxiv preprint doi: https://doi.org/10.1101/2021.02.24.21252329; this version posted February 26, 2021. The copyright holder for this preprint (which was not certified by peer review) is the author/funder, who has granted medRxiv a license to display the preprint in

Long COVID-19 and cognition

\begin{tabular}{|c|c|c|c|c|}
\hline Emotion recognition & GERT & ERQ - Emotion regulation & .33 & .023 \\
\hline & & AMI - Emotional apathy & .28 & .011 \\
\hline & & AMI - Behavioral apathy & .16 & .004 \\
\hline & & Sniff test (anosmia) & .06 & .008 \\
\hline & & AMI - Social apathy & .02 & .027 \\
\hline Anosognosia & Memory dysfunctions & BDI-II - Depression & .62 & $<.001$ \\
\hline & & ISI - Insomnia & .12 & .038 \\
\hline & & AMI - Behavioral apathy & .09 & .040 \\
\hline & & Epworth - Sleepiness & .05 & .033 \\
\hline & Executive functions - Inhibition & AMI - Total apathy & .40 & .011 \\
\hline & Executive functions - Flexibility & AMI - Behavioral score & .28 & .043 \\
\hline & $\begin{array}{l}\text { Executive functions - Working } \\
\text { memory }\end{array}$ & Epworth - Sleepiness & .38 & .015 \\
\hline & & Sniff test (anosmia) & .25 & .015 \\
\hline
\end{tabular}

143 Abbreviations: AMI-behavioral: Apathy Motivation Index - behavioral score (Ang et

144 al., 2017); AMI-emotional: Apathy Motivation Index - emotional score (Ang et al., 2017);

145 AMI-social: Apathy Motivation Index - social score (Ang et al., 2017); AMI-total: Apathy

146 Motivation Index - total score (Ang et al., 2017); BDI-II: Beck Depression Inventory-Second

147 Edition (Beck et al., 1996); BECLA: Batterie d'Evaluation Cognitive du Langage (Macoir et

148 al., 2016); DES: Dissociative Experience Scale (Carlson \& Putnam, 1986); ECS: Emotion

149 Contagion Scale (Doherty, 1997); ERQ: Emotion Regulation Questionnaire (Gross \& John,

150 2003); GERT: Geneva Emotion Recognition Test (Schlegel et al., 2014); Goldberg-Inventory:

151 Goldberg Mania Inventory (Goldberg, 1993); GREFEX: Groupe de Réflexion sur

152 l'Evalutation des Fonctions Exécutives (Roussel \& Godefroy, 2008); MEM III: Wechsler

153 Memory Scale - Third Edition (Drozdick et al., 2018); NV: no variance; $n s$ : not significant;

154 PCL-5: Posttraumatic Stress Disorder Checklist for DSM-5 (Ashbaugh et al., 2016); PSS-14: 
medRxiv preprint doi: https://doi.org/10.1101/2021.02.24.21252329; this version posted February 26, 2021. The copyright holder for this preprint (which was not certified by peer review) is the author/funder, who has granted medRxiv a license to display the preprint in It is made available under a CC-BY-ND 4.0 International license.

Long COVID-19 and cognition

155 Perceived Stress Scale - 14 items (Lesage et al., 2012); Rey figure: Rey-Osterrieth Complex

156 Figure test (Meyers \& Meyers, 1995); RL/RI 16: free/cued recall 16 items (RL/RI-16)

157 (Grober \& Buschke, 1987); SD: standard deviation; STAI-S: State-Trait Anxiety Inventory

158 (Spielberger et al., 1993) STAI-T: State-Trait Anxiety Inventory (Spielberger et al., 1993);

159 TAP: Test for Attentional Performance (TAP), Version 2.1 (Zimmermann \& Fimm, 2007);

160 TMT: Trail Making Test; VOSP: Visual Object and Space Perception battery (Warrington \&

161 James, 1991); WAIS IV: Wechsler Adult Intelligence Scale-Fourth Edition (Wechsler, 2008). 
medRxiv preprint doi: https://doi.org/10.1101/2021.02.24.21252329; this version posted February 26,2021 . The copyright holder for this preprint (which was not certified by peer review) is the author/funder, who has granted medRxiv a license to display the preprint in

Long COVID-19 and cognition

162 DISCUSSION

163 Even though there is growing evidence that SARS-CoV-2 can cause brain damage in the long

164 term, with an impact on cognition even in its mild and moderate forms, to date, the occurrence

165 and nature of such sequelae, the impact of respiratory disease severity in the acute phase, and

166 the relationship between these impairments and psychiatric disorders triggered or exacerbated

167 by the pandemic have not been studied in detail within a single sample of patients. In

168 addition, areas such as instrumental functions (ideomotor praxis, visual perception, or

169 language), cognitive complaints, anosognosia, and emotion recognition following SARS-

$170 \mathrm{CoV}-2$ have yet to be explored. Finally, the relevant medical events have not been controlled

171 in studies published thus far. The present study used a robust, psychometrically validated

172 methodology and a stringent approach to the normative data of neuropsychological tests

173 (excluding borderline scores from the prevalence calculation). We included patients with no

174 history of cancer or neurological and developmental disorders, and no active psychiatric

175 disorders before SARS-CoV-2 infection, and divided them into mild, moderate and severe

176 groups, according to the respiratory severity of the disease during its acute phase.

The present study therefore improves our understanding of what we can call

178 neurological long COVID, highlighting three main patterns of results. First, important

179 prevalence of patients across the three groups performed below the normality threshold in all

180 domains of cognition (except ideomotor praxis) 6-9 months post-infection with SARS-CoV-2.

181 The prevalence of psychiatric symptoms, regardless of disease severity during the acute

182 phase, was also high, and individuals in all three groups exhibited depressive symptoms,

183 anxiety, mania, apathy, stress, PTSD and dissociative disorders, as well as reporting insomnia,

184 fatigue and pathological somnolence. Regarding olfaction, $33.33 \%$ of the mild group, $73.33 \%$

185 of the moderate group, and $46.66 \%$ of the severe group were still hyposmic 6-9 months 
medRxiv preprint doi: https://doi.org/10.1101/2021.02.24.21252329; this version posted February 26, 2021. The copyright holder for this preprint (which was not certified by peer review) is the author/funder, who has granted medRxiv a license to display the preprint in

Long COVID-19 and cognition

186 following infection, and $13.33 \%$ of the severe group were still anosmic. Second, despite the

187 presence of common cognitive deficits across the three groups, some domains of cognition

188 and mood were differentially impacted by the severity of respiratory disease during the acute

189 phase: the severe group performed more poorly than the mild group on long-term episodic

190 memory, and also exhibited more anosognosia for memory dysfunction. The mild group was

191 more depressed, stressed and anxious, and reported more cognitive complaints. Finally, the

192 moderate group recognized multimodal emotions less well than the mild group. All of this had

193 a substantial impact on patients' quality of life. Third, as predicted, neuropsychological

194 deficits correlated with psychiatric disorders such as depressive symptoms, stress and mania,

195 but not all of the variance was explained by psychiatric symptoms or transdiagnostic

196 syndrome (Husain \& Roiser, 2018). A large proportion of the variance was explained by other

197 clinical variables. For instance, the long-term episodic memory deficits displayed by the

198 severe group were positively correlated with emotional apathy, their anosognosia for memory

199 dysfunction was correlated with depression, and their diminished emotion recognition, shared

200 by the moderate group, was positively correlated with hyposmia and/or anosmia.

The present study had several limitations that need to be acknowledged and addressed

202 before we can draw any inferences from our results. The first drawback was a possible

203 recruitment bias. By enrolling volunteers, we may have selected the most severe cases in the

204 mild group (who were interested in the study because of their cognitive complaints), while we

205 may not have recruited the most cognitively affected in the severe group, because they were

206 too disabled to join the study. Second, we had greater proportions of men and diabetics in the

207 severe group. These factors may have had an influence on the cognitive deficits observed in

208 this group, as diabetes is known to impact cognition (McCrimmon, Ryan, \& Frier, 2012), and

209 gender on depression (Spagnolo, Manson, \& Joffe, 2020), with a greater prevalence in women 
medRxiv preprint doi: https://doi.org/10.1101/2021.02.24.21252329; this version posted February 26, 2021. The copyright holder for this preprint (which was not certified by peer review) is the author/funder, who has granted medRxiv a license to display the preprint in

Long COVID-19 and cognition

210 (Mazza et al., 2020). That said, although the proportion of women was higher for both the

211 mild and moderate groups, the mean depression scores by gender in the mild (women: $13.50 \pm$

212 9.10; men: $12.57 \pm 8.52$ ) and moderate (women: $6.11 \pm 5.25$; men: $13.33 \pm 11.25$ ) groups did

213 not indicate a greater proportion of women with depressive symptoms. Third, stroke is more

214 prevalent in patients after a severe SARS-CoV-2 infection (Merkler et al., 2020; Nannoni et

215 al., 2020), and may have gone unseen during the acute phase. In our study, no patient had any

216 central neurological deficit excluding major stroke, but minor stroke cannot be ruled out. Two

217 patients in the severe group reported mild signs of peripheral neuropathy, which may have

218 been due to their diabetes and not a direct consequence of the SARS-CoV-2 infection, while

219 one patient in the severe group had an unstable gait. Fourth, the absence of a control group

220 prevented us from observing a possible general effect of the pandemic and the resulting public

221 health measures on mental health. In the present study, the analyses of prevalence were based

222 on standardized normative data, allowing us to run comparisons with the normal population.

223 The tests were chosen carefully for their psychometric validity, with adequate sensitivity and

224 specificity. It is interesting to note that in a recent study, our multimodal emotion recognition

225 task (GERT) was administered to 469 participants during the pandemic (Schlegel, von

226 Gugelberg, Makowski, Gubler, \& Troche, 2021) but the authors failed to find a reduction in

227 performances compared with validation studies (Schlegel et al., 2014), reinforcing the

228 hypothesis that our results reflected a specific effect of the infection and not just the public

229 health context. Fifth and last, it is important to note the relatively small number of

230 participants, which prevented us from considering more covariates. Nevertheless, the power

231 analysis, based on a previous study of the neurocognitive effects of SARS-CoV-2, did allow

232 us to estimate the necessary sample size. 
medRxiv preprint doi: https://doi.org/10.1101/2021.02.24.21252329; this version posted February 26, 2021. The copyright holder for this preprint (which was not certified by peer review) is the author/funder, who has granted medRxiv a license to display the preprint in

Long COVID-19 and cognition

The present results demonstrate that cognitive deficits can be observed 6-9 months post-SARS-CoV-2 infection, regardless of the severity of the disease in the acute phase. They corroborate previous observations for the executive, attentional and memory domains, and go one step further, with exhaustive neuropsychological and psychiatric assessments demonstrating impairments in other previously unexplored cognitive and psychiatric domains. Impairments were evident not only in the severe patient group, but also in the moderate and mild groups. These deficits had an impact on quality of life, notably in the mild patients, as evidenced by our results. These findings could be of great importance in understanding the

241 long-term damage and consequences of SARS-CoV-2 infection on cognition and mental

242 health. The relatively high prevalence of certain cognitive and psychiatric disorders,

243 regardless of the severity of the disease in the acute phase, suggests that long-term patient 244 management following SARS-CoV-2 infection may need to be adapted. Importantly, the 245 etiology of these disorders needs to be established, in order to provide people experiencing 246 these long-term sequelae with the best possible care. One potential explanation for these 247 effects, based on observational studies of the psychiatric impact of the pandemic in the 248 general population (Bäuerle et al., 2020), is that these cognitive deficits result from a stressful 249 or traumatic context. In this case, specific interventions on certain psychiatric variables could considerably reduce their long-term impact on cognition and improve daily functioning.

251 Nevertheless, the present results do not exclude the hypothesis of direct damage of brain 252 networks by SARS-CoV-2 and its neurotropism, as well as indirect neurobiological effects, 253 which could lead to both to psychiatric and neurological disorders. COVID-19 may induce 254 CNS disturbance, and four main pathogenic mechanisms may act in combination: i) direct 255 viral encephalitis, ii) systemic inflammation, iii) peripheral organ dysfunction (liver, kidney, 256 lung), and iv) cerebrovascular changes (Iadecola, Anrather, \& Kamel, 2020). At this stage, it 
medRxiv preprint doi: https://doi.org/10.1101/2021.02.24.21252329; this version posted February 26, 2021. The copyright holder for this preprint (which was not certified by peer review) is the author/funder, who has granted medRxiv a license to display the preprint in

Long COVID-19 and cognition

257 is difficult to determine whether the cognitive deficits can be regarded as a marker of brain 258 damage, and/or should be linked to psychiatric variables that may themselves result directly 259 from infection with SARS-CoV-2 or else be triggered by the stressful nature of the general 260 pandemic and the individual experience of the disease.

Second, this study highlighted the presence of differential cognitive and psychiatric

262 profiles at 6-9 months post-infection as a function of the respiratory severity of the SARS-

263 CoV-2 infection in the acute phase. This suggests the existence of different clinical

264 phenotypes. In the identification/discrimination of these phenotypes, different cognitive

265 variables seem to be of interest, starting with cognitive complaints and anosognosia. While

266 the severe patients exhibited anosognosia for their memory dysfunction and greater long-term

267 verbal memory impairment than the mild patients did, the latter had more cognitive

268 complaints. This fits in well with the observations of Almeria et al. (2020), who found that the

269 patients with the most serious cognitive complaints did not have significantly more

270 neuropsychological impairments. In this sense, the tendency of the severe patients to report

271 greater wellbeing in quality-of-life assessments, together with the lack of awareness of their

272 cognitive difficulties, may be a clinical characteristic to bear in mind when interviewing this

273 type of patient. The present results in the domain of emotion recognition and episodic

274 memory are also highly relevant to the current debate on the neurotropism of SARS-CoV-2.

275 One of the main hypotheses regarding the pathways of direct attack of the CNS assumes

276 olfactory transmucosal invasion by the virus (Meinhardt et al., 2021). This hypothesis appears

277 to be supported by our results. It is worth noting that episodic memory and emotion

278 recognition were identified in a PCA as variables that explained most of the variance of our

279 data, and this first component was significantly correlated with hyposmia/anosmia, in addition

280 to stress and emotional apathy. Interestingly, a recent ${ }^{18}$ F-FDG PET study demonstrated 
medRxiv preprint doi: https://doi.org/10.1101/2021.02.24.21252329; this version posted February 26, 2021. The copyright holder for this preprint (which was not certified by peer review) is the author/funder, who has granted medRxiv a license to display the preprint in

Long COVID-19 and cognition

281 hypometabolism at about 8 weeks post-infection in brain regions common to emotion and

282 olfaction in patients with SARS-CoV-2 (Guedj et al., 2020). Moreover, the literature suggests

283 that the viral load was probably greater in our severe group (Fajnzylber et al., 2020; Magleby

284 et al., 2020), which may have contributed to stronger effects on olfaction and emotion

285 recognition. This pathway could also partially explain the psychiatric results via disruption of

286 the limbic network, including subcortical regions (Lane, 2008), by SARS-CoV-2.

Our third level of analysis enabled us to go further in characterizing the hypothesized

clinical phenotypes. Quantified results pointed to the presence of at least three profiles

(patient clusters), corroborating the clinical impressions we had when interviewing and

assessing the patients for this study. Patients with the first (neurological) profile were

typically aged about 55 years, mostly men, of average educational level, and a small

proportion of them had a history of diabetes, cardiovascular disease, or sleep apnea syndrome.

At the cognitive level, these patients displayed long-term memory, executive and language

disorders. They had more severe anosognosia for their memory difficulties. Nearly all of them

were aged about 45-50 years, and there were equal numbers of men and women. No

297 significant medical antecedents were noted, and the majority of them had had mild or

moderate respiratory disease. At the cognitive level, they displayed executive and attentional

301 stress, and more sporadically exhibited PTSD and dissociative disorders. Our results also

302 indicated the presence of a third (mixed) profile combining the symptoms and clinical 303 characteristics of the two previously described profiles. 
medRxiv preprint doi: https://doi.org/10.1101/2021.02.24.21252329; this version posted February 26, 2021. The copyright holder for this preprint (which was not certified by peer review) is the author/funder, who has granted medRxiv a license to display the preprint in It is made available under a CC-BY-ND 4.0 International license .

Long COVID-19 and cognition

\section{CONCLUSION}

306 This study unambiguously demonstrates the presence of long-term neuropsychological

307 sequelae following SARS-CoV-2 infection, regardless of the severity of the respiratory

308 disease in the acute phase. Some of the cognitive deficits could be explained by psychiatric

309 variables, emphasizing the importance of considering a broad range of psychiatric symptoms.

310 However, not all neuropsychological sequelae could be explained by these variables. The

311 presence of correlations between olfaction, emotion recognition and episodic memory, which

312 share common functional and anatomical substrates, reinforces the hypothesis that the virus

313 targets the CNS (and notably the limbic system). Finally, the data support the notion of

314 different clinical phenotypes, paving the way for clinical guidelines and recommendations for

315 the management of long-term neurological impairment following SARS-CoV-2 infection. 
medRxiv preprint doi: https://doi.org/10.1101/2021.02.24.21252329; this version posted February 26, 2021. The copyright holder for this preprint (which was not certified by peer review) is the author/funder, who has granted medRxiv a license to display the preprint in It is made available under a CC-BY-ND 4.0 International license.

Long COVID-19 and cognition

\section{ACKNOWLEDGEMENTS}

The present research was supported by the Swiss National Science Foundation (SNSF)

318 within the framework of the COVID-19 National Research Program (NRP 78; grant no.

319 407840_198438, RNP 78) to JAP (PI) and FA (Co-PI). The funders had no role in data

320 collection, discussion of content, preparation of the manuscript, or decision to publish. We

321 would like to thank the patients for contributing their time to this study.

CONFLICT OF INTEREST

The authors report no conflicts of interest. 
medRxiv preprint doi: https://doi.org/10.1101/2021.02.24.21252329; this version posted February 26, 2021. The copyright holder for this preprint (which was not certified by peer review) is the author/funder, who has granted medRxiv a license to display the preprint in

Long COVID-19 and cognition

\section{REFERENCES}

Almeria, M., Cejudo, J., Sotoca, J., Deus, J., \& Krupinski, J. (2020). Cognitive profile following COVID-19 infection: Clinical predictors leading to neuropsychological impairment. Brain, behavior, \& immunity-health, 9, 100163.

Ang, Y.-S., Lockwood, P., Apps, M. A., Muhammed, K., \& Husain, M. (2017). Distinct subtypes of apathy revealed by the apathy motivation index. PLoS One, 12(1), e0169938.

Ashbaugh, A. R., Houle-Johnson, S., Herbert, C., El-Hage, W., \& Brunet, A. (2016). Psychometric validation of the English and French versions of the Posttraumatic Stress Disorder Checklist for DSM-5 (PCL-5). PloS one, 11(10).

Baig, A. M., Khaleeq, A., Ali, U., \& Syeda, H. (2020). Evidence of the COVID-19 virus targeting the CNS: tissue distribution, host-virus interaction, and proposed neurotropic mechanisms. ACS chemical neuroscience, 11(7), 995-998.

Bäuerle, A., Teufel, M., Musche, V., Weismüller, B., Kohler, H., Hetkamp, M., . . Skoda, E.M. (2020). Increased generalized anxiety, depression and distress during the COVID19 pandemic: a cross-sectional study in Germany. Journal of Public Health, 42(4), $672-678$.

Beck, A. T., Steer, R. A., \& Brown, G. K. (1996). Manual for the beck depression inventoryII. San Antonio, TX: Psychological Corporation, 1, 82.

Bousquet, J., Bullinger, M., Fayol, C., Marquis, P., Valentin, B., \& Burtin, B. (1994). Assessment of quality of life in patients with perennial allergic rhinitis with the French version of the SF-36 Health Status Questionnaire. Journal of allergy and clinical immunology, 94(2), 182-188. 
medRxiv preprint doi: https://doi.org/10.1101/2021.02.24.21252329; this version posted February 26, 2021. The copyright holder for this preprint (which was not certified by peer review) is the author/funder, who has granted medRxiv a license to display the preprint in

Long COVID-19 and cognition

Brann, D. H., Tsukahara, T., Weinreb, C., Lipovsek, M., Van den Berge, K., Gong, B., . . . Fletcher, R. B. (2020). Non-neuronal expression of SARS-CoV-2 entry genes in the olfactory system suggests mechanisms underlying COVID-19-associated anosmia. Science advances, 6(31), eabc5801.

Butowt, R., \& Bilinska, K. (2020). SARS-CoV-2: olfaction, brain infection, and the urgent need for clinical samples allowing earlier virus detection. ACS chemical neuroscience, 11(9), 1200-1203.

Carlson, E. B., \& Putnam, F. W. (1986). Dissociative experiences scale. Journal of Nervous and Mental Disease, 174(12).

Cascella, M., Di Napoli, R., Carbone, D., Cuomo, G. F., Bimonte, S., \& Muzio, M. R. (2018). Chemotherapy-related cognitive impairment: mechanisms, clinical features and research perspectives. Recenti progressi in medicina, 109(11), 523-530.

De Maria, A., Varese, P., Dentone, C., Barisione, E., \& Bassetti, M. (2020). High prevalence of olfactory and taste disorder during SARS-CoV-2 infection in outpatients. Journal of medical virology, 92(11), 2310-2311.

Debouverie, M., Pittion-Vouyovitch, S., Louis, S., \& Guillemin, F. (2007). Validity of a French version of the fatigue impact scale in multiple sclerosis. Multiple Sclerosis Journal, 13(8), 1026-1032.

Doherty, R. W. (1997). The emotional contagion scale: A measure of individual differences. Journal of nonverbal Behavior, 21(2), 131-154.

Doty, R. L. (2008). The olfactory vector hypothesis of neurodegenerative disease: is it viable? Annals of Neurology: Official Journal of the American Neurological Association and the Child Neurology Society, 63(1), 7-15. 
medRxiv preprint doi: https://doi.org/10.1101/2021.02.24.21252329; this version posted February 26, 2021. The copyright holder for this preprint (which was not certified by peer review) is the author/funder, who has granted medRxiv a license to display the preprint in

Long COVID-19 and cognition

373 Drozdick, L. W., Raiford, S. E., Wahlstrom, D., \& Weiss, L. G. (2018). The Wechsler Adult Intelligence Scale_-Fourth Edition and the Wechsler Memory Scale-Fourth Edition.

Fajnzylber, J., Regan, J., Coxen, K., Corry, H., Wong, C., Rosenthal, A., . . Atyeo, C. mortality. Nature communications, 11(1), 1-9.

Fodoulian, L., Tuberosa, J., Rossier, D., Boillat, M., Kan, C., Pauli, V., . . . Carleton, A. (2020). Sars-cov-2 receptors and entry genes are expressed in the human olfactory neuroepithelium and brain. Iscience, 23(12), 101839.

Frei, A., Balzer, C., Gysi, F., Leros, J., Plohmann, A., \& Steiger, G. (2016). Kriterien zur Zuordnungen zur Funktions-und Arbeitsfähigkeit. Zeitschrift für Neuropsychologie.

Goldberg, I. K. (1993). Questions \& Answers about Depression and its Treatment: a consultation with a leading psychiatrist: Charles PressPub.

Grober, E., \& Buschke, H. (1987). Genuine memory deficits in dementia. Dev. Neuropsychol, $3,13-36$.

Gross, J., \& John, O. (2003). Emotion regulation questionnaire. NeuroImage, 48(10), 9-9.

Guedj, Campion, J., Dudouet, P., Kaphan, E., Bregeon, F., Tissot-Dupont, H., . . Ceccaldi, M. (2021). 18 F-FDG brain PET hypometabolism in patients with long COVID. European journal of nuclear medicine and molecular imaging, 1-11.

392 Guedj, Million, M., Dudouet, P., Tissot-Dupont, H., Bregeon, F., Cammilleri, S., \& Raoult, D. (2020). 18 F-FDG brain PET hypometabolism in post-SARS-CoV-2 infection: substrate for persistent/delayed disorders? European journal of nuclear medicine and molecular imaging, 1-4. 
medRxiv preprint doi: https://doi.org/10.1101/2021.02.24.21252329; this version posted February 26, 2021. The copyright holder for this preprint (which was not certified by peer review) is the author/funder, who has granted medRxiv a license to display the preprint in

Long COVID-19 and cognition

396 Hampshire, A., Trender, W., Chamberlain, S., Jolly, A., Grant, J. E., Patrick, F., .. . Hellyer, P. (2020). Cognitive deficits in people who have recovered from COVID-19 relative to controls: An N=84,285 online study. MedRxiv.

Heaton, R. K., Grant, I., \& Matthews, C. G. (1991). Comprehensive norms for an expanded Halstead-Reitan battery: demographic corrections, research findings, and clinical applications; with a supplement for the Wechsler Adult Intelligence Scale-Revised (WAIS-R): Psychological Assessment Resources.

404

Husain, M., \& Roiser, J. P. (2018). Neuroscience of apathy and anhedonia: a transdiagnostic approach. Nature Reviews Neuroscience, 19(8), 470-484.

406

Iadecola, C., Anrather, J., \& Kamel, H. (2020). Effects of COVID-19 on the nervous system. Cell.

Johns, M. W. (1991). A new method for measuring daytime sleepiness: the Epworth sleepiness scale. sleep, 14(6), 540-545.

Kandemirli, S. G., Altundag, A., Yildirim, D., Sanli, D. E. T., \& Saatci, O. (2021). Olfactory Bulb MRI and paranasal sinus CT findings in persistent COVID-19 anosmia. Academic Radiology, 28(1), 28-35.

Kessels, R. P., Van Zandvoort, M. J., Postma, A., Kappelle, L. J., \& De Haan, E. H. (2000). The Corsi block-tapping task: standardization and normative data. Applied neuropsychology, 7(4), 252-258.

Kohler, O., Krogh, J., Mors, O., \& Eriksen Benros, M. (2016). Inflammation in depression and the potential for anti-inflammatory treatment. Current neuropharmacology, 14(7), 732-742.

Landis, B. N., Vodicka, J., \& Hummel, T. (2010). Olfactory dysfunction following herpetic meningoencephalitis. Journal of neurology, 257(3), 439-443. 
medRxiv preprint doi: https://doi.org/10.1101/2021.02.24.21252329; this version posted February 26, 2021. The copyright holder for this preprint (which was not certified by peer review) is the author/funder, who has granted medRxiv a license to display the preprint in

Long COVID-19 and cognition

420 Lane, R. D. (2008). Neural substrates of implicit and explicit emotional processes: a unifying framework for psychosomatic medicine. Psychosomatic medicine, 70(2), 214-231.

Lee, J.-M., \& Lee, S. J. (2020). Olfactory and gustatory dysfunction in a COVID-19 patient with ankylosing spondylitis treated with etanercept: case report. Journal of Korean medical science, 35(21).

Lehmann, E. L. (2012). Parametric versus nonparametrics: two alternative methodologies. In Selected works of EL Lehmann (pp. 437-445): Springer.

Leicht, H., Berwig, M., \& Gertz, H.-J. (2010). Anosognosia in Alzheimer's disease: the role of impairment levels in assessment of insight across domains. Journal of the International Neuropsychological Society: JINS, 16(3), 463.

Lesage, F.-X., Berjot, S., \& Deschamps, F. (2012). Psychometric properties of the French versions of the Perceived Stress Scale. International journal of occupational medicine and environmental health, 25(2), 178-184.

Macoir, J., Gauthier, C., Jean, C., \& Potvin, O. (2016). BECLA, a new assessment battery for acquired deficits of language: Normative data from Quebec-French healthy younger and older adults. Journal of the neurological sciences, 361, 220-228.

Magleby, R., Westblade, L. F., Trzebucki, A., Simon, M. S., Rajan, M., Park, J., . . Satlin, M. J. (2020). Impact of SARS-CoV-2 viral load on risk of intubation and mortality among hospitalized patients with coronavirus disease 2019. Clinical infectious diseases.

Mahieux-Laurent, F., Fabre, C., Galbrun, E., Dubrulle, A., Moroni, C., \& Sud, G. d. r. s. 1. p. d. C. I.-d.-F. (2009). Validation d'une batterie brève d'évaluation des praxies gestuelles pour consultation Mémoire. Évaluation chez 419 témoins, 127 patients 
medRxiv preprint doi: https://doi.org/10.1101/2021.02.24.21252329; this version posted February 26, 2021. The copyright holder for this preprint (which was not certified by peer review) is the author/funder, who has granted medRxiv a license to display the preprint in

Long COVID-19 and cognition

atteints de troubles cognitifs légers et 320 patients atteints d'une démence. Revue neurologique, 165(6-7), 560-567.

Mao, L., Jin, H., Wang, M., Hu, Y., Chen, S., He, Q., . . Wang, D. (2020). Neurologic manifestations of hospitalized patients with coronavirus disease 2019 in Wuhan, China. JAMA neurology, 77(6), 683-690.

Mazza, M. G., De Lorenzo, R., Conte, C., Poletti, S., Vai, B., Bollettini, I., . . RovereQuerini, P. (2020). Anxiety and depression in COVID-19 survivors: Role of inflammatory and clinical predictors. Brain, behavior, and immunity, 89, 594-600.

McCrimmon, R. J., Ryan, C. M., \& Frier, B. M. (2012). Diabetes and cognitive dysfunction. The Lancet, 379(9833), 2291-2299.

Meinhardt, J., Radke, J., Dittmayer, C., Franz, J., Thomas, C., Mothes, R., . . Greuel, S. (2021). Olfactory transmucosal SARS-CoV-2 invasion as a port of central nervous system entry in individuals with COVID-19. Nature neuroscience, 24(2), 168-175.

Merkler, A. E., Parikh, N. S., Mir, S., Gupta, A., Kamel, H., Lin, E., . . Bruce, S. S. (2020). Risk of ischemic stroke in patients with coronavirus disease 2019 (COVID-19) vs patients with influenza. JAMA neurology, 77(11), 1366-1372.

Meyers, J. E., \& Meyers, K. R. (1995). Rey Complex Figure Test and recognition trial professional manual: Psychological Assessment Resources.

461 Miyake, A., Friedman, N. P., Emerson, M. J., Witzki, A. H., Howerter, A., \& Wager, T. D. (2000). The unity and diversity of executive functions and their contributions to complex "frontal lobe" tasks: A latent variable analysis. Cognitive psychology, 41(1), 49-100.

Morin, C. M. (1993). Insomnia: psychological assessment and management: Guilford press. 
medRxiv preprint doi: https://doi.org/10.1101/2021.02.24.21252329; this version posted February 26, 2021. The copyright holder for this preprint (which was not certified by peer review) is the author/funder, who has granted medRxiv a license to display the preprint in

Long COVID-19 and cognition

466 Nannoni, S., de Groot, R., Bell, S., \& Markus, H. S. (2020). Stroke in COVID-19: a systematic review and meta-analysis. International Journal of Stroke, 1747493020972922

Oldfield, R. C. (1971). The assessment and analysis of handedness: the Edinburgh inventory. Neuropsychologia, 9(1), 97-113.

Oxley, T. J., Mocco, J., Majidi, S., Kellner, C. P., Shoirah, H., Singh, I. P., . . Yaeger, K. A. (2020). Large-vessel stroke as a presenting feature of Covid-19 in the young. New England Journal of Medicine, 382(20), e60.

Piras, M. R., Magnano, I., Canu, E. D. G., Paulus, K. S., Satta, W. M., Soddu, A., . . Aiello, neuropsychological, neuroradiological, and neurophysiological findings. Journal of Neurology, Neurosurgery \& Psychiatry, 74(7), 878-885.

Regina, J., Papadimitriou-Olivgeris, M., Burger, R., Le Pogam, M.-A., Niemi, T., Filippidis, P., . . Kampouri, E. (2020). Epidemiology, risk factors and clinical course of SARSCoV-2 infected patients in a Swiss university hospital: an observational retrospective study. PLoS One, 15(11), e0240781.

Rogers, J. P., Chesney, E., Oliver, D., Pollak, T. A., McGuire, P., Fusar-Poli, P., . . David, coronavirus infections: a systematic review and meta-analysis with comparison to the COVID-19 pandemic. The Lancet Psychiatry.

Röhr, S., Müller, F., Jung, F., Apfelbacher, C., Seidler, A., \& Riedel-Heller, S. G. (2020). Psychosocial impact of quarantine measures during serious coronavirus outbreaks: a rapid review. Psychiatrische Praxis, 47(4), 179-189. 
medRxiv preprint doi: https://doi.org/10.1101/2021.02.24.21252329; this version posted February 26, 2021. The copyright holder for this preprint (which was not certified by peer review) is the author/funder, who has granted medRxiv a license to display the preprint in

Long COVID-19 and cognition

Rosen, H. J., Alcantar, O., Rothlind, J., Sturm, V., Kramer, J. H., Weiner, M., \& Miller, B. L. (2010). Neuroanatomical correlates of cognitive self-appraisal in neurodegenerative disease. NeuroImage, 49(4), 3358-3364.

Roth, R. M., Gioia, G. A., \& Isquith, P. K. (2005). BRIEF-A: Behavior Rating Inventory of

Roussel, M., \& Godefroy, O. (2008). La batterie GREFEX: données normatives. Fonctions exécutives et pathologies neurologiques et psychiatriques, 231-252.

Salari, N., Hosseinian-Far, A., Jalali, R., Vaisi-Raygani, A., Rasoulpoor, S., Mohammadi, M., ... Khaledi-Paveh, B. (2020). Prevalence of stress, anxiety, depression among the general population during the COVID-19 pandemic: a systematic review and metaanalysis. Globalization and health, 16(1), 1-11.

500 Schlegel, K., Grandjean, D., \& Scherer, K. R. (2014). Introducing the Geneva emotion recognition test: an example of Rasch-based test development. Psychological Assessment, 26(2), 666.

Schlegel, K., von Gugelberg, H. M., Makowski, L. M., Gubler, D. A., \& Troche, S. J. (2021). Emotion Recognition Ability as a Predictor of Well-Being During the COVID-19 Pandemic. Social Psychological and Personality Science, 1948550620982851.

506 Soudry, Y., Lemogne, C., Malinvaud, D., Consoli, S.-M., \& Bonfils, P. (2011). Olfactory system and emotion: common substrates. European annals of otorhinolaryngology, head and neck diseases, 128(1), 18-23. the COVID-19 pandemic can teach us. In: American College of Physicians. 
medRxiv preprint doi: https://doi.org/10.1101/2021.02.24.21252329; this version posted February 26, 2021. The copyright holder for this preprint (which was not certified by peer review) is the author/funder, who has granted medRxiv a license to display the preprint in

Long COVID-19 and cognition

511 Spielberger, C. D., Gorsuch, R. L., Lushene, R., Vagg, P. R., \& Jacobs, G. A. (1993). Manuel de l'inventaire d'anxiété état-trait forme Y (STAI-Y) [Inventory of state-trait anxiety manual]. Paris: Editions du Centre de Psychologie Appliquée.

Thomas-Antérion, C., Ribas, C., Honoré-Masson, S., Million, J., \& Laurent, B. (2004). anxiodépressifs et de témoins avec le QPC (Questionnaire de Plainte Cognitive). NPG Neurologie-Psychiatrie-Gériatrie, 4(20), 30-34.

Tondelli, M., Barbarulo, A. M., Vinceti, G., Vincenzi, C., Chiari, A., Nichelli, P. F., \& Zamboni, G. (2018). Neural correlates of Anosognosia in Alzheimer's disease and mild cognitive impairment: A multi-method assessment. Frontiers in Behavioral Neuroscience, 12, 100.

Troyer, E. A., Kohn, J. N., \& Hong, S. (2020). Are we facing a crashing wave of neuropsychiatric sequelae of COVID-19? Neuropsychiatric symptoms and potential

Vaira, L. A., Hopkins, C., Sandison, A., Manca, A., Machouchas, N., Turilli, D., . . Cossu, A. (2020). Olfactory epithelium histopathological findings in long-term coronavirus disease 2019 related anosmia. The Journal of Laryngology \& Otology, 1-5.

Van der Linden, M., Coyette, F., Poitrenaud, J., Kalafat, M., Calicis, F., Wyns, C., \& Adam, S. (2004). II. L'épreuve de rappel libre/rappel indicé à 16 items (RL/RI-16). ... Smitt, P. A. S. (2016). Anti-LGI1 encephalitis: clinical syndrome and long-term follow-up. Neurology, 87(14), 1449-1456. 
medRxiv preprint doi: https://doi.org/10.1101/2021.02.24.21252329; this version posted February 26, 2021. The copyright holder for this preprint (which was not certified by peer review) is the author/funder, who has granted medRxiv a license to display the preprint in It is made available under a CC-BY-N

Long COVID-19 and cognition

533 Varatharaj, A., Thomas, N., Ellul, M. A., Davies, N. W., Pollak, T. A., Tenorio, E. L., .. . Zandi, M. (2020). Neurological and neuropsychiatric complications of COVID-19 in 153 patients: a UK-wide surveillance study. The Lancet Psychiatry, 7(10), 875-882.

Wang, C., Pan, R., Wan, X., Tan, Y., Xu, L., Ho, C. S., \& Ho, R. C. (2020). Immediate psychological responses and associated factors during the initial stage of the 2019 coronavirus disease (COVID-19) epidemic among the general population in China. International journal of environmental research and public health, 17(5), 1729.

540 Warrington, E. K., \& James, M. (1991). The visual object and space perception battery.

541 Wechsler, D. (2008). Wechsler adult intelligence scale-Fourth Edition (WAIS-IV). San Antonio, TX: NCS Pearson, 22(498), 1.

Wendelken, L. A., \& Valcour, V. (2012). Impact of HIV and aging on neuropsychological function. Journal of neurovirology, 18(4), 256-263.

Woo, M. S., Malsy, J., Pöttgen, J., Seddiq Zai, S., Ufer, F., Hadjilaou, A., . . . Heesen, C. (2020). Frequent neurocognitive deficits after recovery from mild COVID-19. Brain Communications, 2(2), fcaa205. 
medRxiv preprint doi: https://doi.org/10.1101/2021.02.24.21252329; this version posted February 26, 2021. The copyright holder for this preprint (which was not certified by peer review) is the author/funder, who has granted medRxiv a license to display the preprint in It is made available under a CC-BY-ND 4.0 International license .

Long COVID-19 and cognition

562 Rey Figure - Copy score

563 Rey Figure - Immediate recall (3')

564 Rey Figure - Delayed recall (20')

565 Stroop (GREFEX)- Interference - Errors

566 Stroop (GREFEX)- Interference/naming - Score

567 TMT B-A (GREFEX) - Score

568 Verbal fluency (GREFEX) - Literal (2')

569 Verbal fluency (GREFEX) - Categorical (2')

570 TAP phasic alertness - Without warning sound - Reaction time

571 TAP phasic alertness - With warning sound - Reaction time

572 TAP phasic alertness - Alertness index

573 TAP sustained attention - Item omissions

574 TAP sustained attention - False alarm

575 TAP divided attention - Audio condition - Reaction time

576 TAP divided attention - Visual condition - Reaction time

577 TAP divided attention - Total omissions

578 TAP divided attention - Total false alarms

579 TAP Incompatibility task - Visual fields * Hands score

580 BECLA - Semantic image matching

581 BECLA - Semantic word matching 
medRxiv preprint doi: https://doi.org/10.1101/2021.02.24.21252329; this version posted February 26, 2021. The copyright holder for this preprint (which was not certified by peer review) is the author/funder, who has granted medRxiv a license to display the preprint in It is made available under a CC-BY-ND 4.0 International license.

Long COVID-19 and cognition

582 BECLA - Object and action image naming

583 BECLA - Word repetition

584 BECLA - Nonword repetition

585 Evaluation of gestural praxis - Symbolic gestures

586 Evaluation of gestural praxis - Action pantomimes

587 Evaluation of gestural praxis - Meaningless gestures

588 VOSP - Fragmented letters

589 VOSP - Object decision

590 VOSP - Number localization

591 VOSP - Cubic counting

592 WAIS IV - Puzzle

593 WAIS IV - Matrix

594 GERT - Emotion recognition task

595 Anosognosia - Memory functions

596 Anosognosia - Executive functions - Working memory

597 Anosognosia - Executive functions - Inhibition

598 Anosognosia - Executive functions - Flexibility 